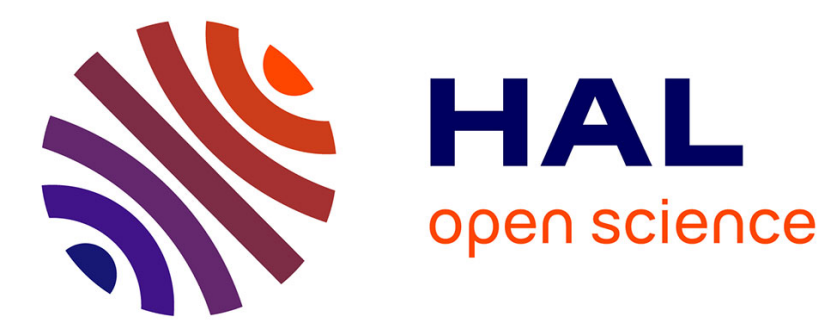

\title{
On the propagation of camera sensor noise to displacement maps obtained by DIC - an experimental study
}

Benoît Blaysat, Michel Grediac, Frédéric Sur

\section{- To cite this version:}

Benoît Blaysat, Michel Grediac, Frédéric Sur. On the propagation of camera sensor noise to displacement maps obtained by DIC - an experimental study. Experimental Mechanics, 2016, 56 (6), pp.919-944. 10.1007/s11340-016-0130-9 . hal-01269655

\section{HAL Id: hal-01269655 \\ https://hal.inria.fr/hal-01269655}

Submitted on 12 Feb 2016

HAL is a multi-disciplinary open access archive for the deposit and dissemination of scientific research documents, whether they are published or not. The documents may come from teaching and research institutions in France or abroad, or from public or private research centers.
L'archive ouverte pluridisciplinaire HAL, est destinée au dépôt et à la diffusion de documents scientifiques de niveau recherche, publiés ou non, émanant des établissements d'enseignement et de recherche français ou étrangers, des laboratoires publics ou privés. 


\title{
On the propagation of camera sensor noise to displacement maps obtained by DIC - an experimental study
}

\author{
Benoît Blaysat, Michel GréDiac, Frédéric SuR ${ }^{\ddagger}$
}

\begin{abstract}
This paper focuses on one of the metrological properties of DIC, namely displacement resolution. More specifically, the study aims to validate, in the environment of an experimental mechanics laboratory, a recent generalized theoretical prediction of displacement resolution. Indeed, usual predictive formulas available in the literature neither take into account sub-pixel displacement, nor have been validated in an experimental mechanics laboratory environment, nor are applicable to all types of DIC (Global as well as Local). Here, the formula used to account for sub-pixel displacements is first recalled, and an accurate model of the sensor noise is introduced. The hypotheses required for the elaboration of this prediction are clearly stated. The formula is then validated using experimental data. Since rigid body motion between the specimen and the camera impairs the experimental data, and since sensor noise is signal-dependent, particular tools need to be introduced in order to ensure the consistency between the observed image noise and the model on which prediction hypotheses are based. Pre-processing tools introduced for another full-field measurement approach, namely the Grid Method, are employed to address these issues.
\end{abstract}

Keywords: Digital Image Correlation, Displacement maps, Generalized Anscombe transform, Measurement resolution, Micro-movements, Noise, Resolution prediction

This is the author-manuscript version of:

B. Blaysat, M. Grédiac, F. Sur. On the propagation of camera sensor noise to displacement maps obtained by DIC - an experimental study. Experimental Mechanics, Springer, 2016.

DOI: $10.1007 / \mathrm{s} 11340-016-0130-9$

\footnotetext{
${ }^{*}$ Corresponding author. Clermont Université, Université Blaise Pascal, Institut Pascal, UMR CNRS 6602, BP 10448, 63000 Clermont-Ferrand, France

${ }^{\dagger}$ Clermont Université, Université Blaise Pascal, Institut Pascal, UMR CNRS 6602, BP 10448, 63000 ClermontFerrand, France

${ }^{\ddagger}$ Laboratoire Lorrain de Recherche en Informatique et ses Applications, UMR CNRS 7503, Université de Lorraine, CNRS, INRIA projet Magrit, Campus Scientifique, BP 239, 54506 Vandœuvre-lès-Nancy Cedex, France
} 


\section{Introduction}

One of the goals of experimental mechanics is to observe physical material deformations during testing in order to both study the material itself and characterize existing models. Observations during testing have radically changed in the last decades thanks to the rise of full-field measurement techniques. These techniques, such as Digital Image Correlation (DIC), do not only allow the observation of a set of a few points, but also offer the direct estimation of fields of mechanical information such as displacement maps. In experimental mechanics, DIC was introduced by Sutton in the $1980 \mathrm{~s}[8,46]$. It is nowadays widely used in the experimental mechanics community, because commercial packages are relatively affordable and also because of the richness of the information it provides. The technique itself is still a subject of development, and novel methods have been proposed. For instance, from the original subset-based DIC, namely Local-DIC (L-DIC), Finite-Element-based and later on handbook-functions-based DIC, namely Global-DIC (G-DIC), have emerged [2] and are still being studied [32, 31]. The a priori definition of the kinematics has improved the robustness of the technique, and can be easily associated with characterization procedures, since dedicated kinematics can be chosen; see [37, 38] and more recently $[23,5]$.

Displacement maps are usual DIC outputs. Such quantities are directly used for observation purposes $(e . g .,[17])$, or are post-processed to characterize constitutive materials (e.g., [3]). It is worth mentioning that any measurement tool, especially in the field of mechanical engineering, begins to be well-founded as soon as its metrological performance is also known.

In [20], the mechanical performance of a measurement tool is defined through three quantities: bias, spatial resolution and measurement resolution. Since these quantities are highly correlated, any detailed study of the metrological performance as a whole first requires that we focus on each of them. In the present study, only one of the main parameters characterizing this performance is studied: measurement resolution. This is a challenge as such, so bias and spatial resolution notions are deliberately not tackled. Measurement resolution is defined as "the smallest change in a quantity being measured that causes a perceptible change in the corresponding indication" [20]; in other words, this is the smallest quantity that reliably emerges from the noise floor. In this study, the noise floor is considered as being caused by sensor noise only. The effects of outof-plane motion, lens distortion or aberrations are consequently not studied in this article; for further information, see $[52,45,29,30]$. For the displacement maps studied here, we thus assume the measurement resolution to be equal to the standard deviation of the noise that corrupts the displacement, as suggested in [13].

In parallel with DIC developments, the measurement resolution of full-field outputs has thus begun to be studied during the past decade (e.g., [50, 40, 48, 21, 49, 47] for L-DIC or [2, 39, 35, $36,18]$ for G-DIC). These studies present intrinsic and extrinsic parameters of DIC techniques which affect displacement resolution. Pattern quality and camera sensor noise are among the parameters impairing the resolution of the DIC output. The pattern is pictured before and during testing, and therefore carries the information that requires post-processing in order to obtain displacement maps. It thus plays a crucial rule in the determination of displacement resolution [22]. Optimized patterns can even be designed [6] for this purpose. Of course, the signal that the camera sensor provides contains noise. This noise has been thoroughly studied in the image processing community [19].

The propagation of camera sensor noise to displacement maps obtained using DIC, and consequently the elaboration of a predictive formula for displacement resolution, is the purpose of the present paper. More specifically, we are interested in the validation of a predictive formula in an experimental mechanics laboratory environment rather than in the study of the resolution itself and its dependence on pattern quality, subset size, or other extrinsic parameters of the DIC 
solving scheme.

For G-DIC, predictive formulas for displacement resolution already exist in the literature. First, an estimate of the average displacement resolution over the observed zone was proposed, e.g. $[2,39]$. Later on, local predictions of the resolution were introduced; see for instance $[35,36,18,4]$. However, their validation using experimental data has not been carefully studied. The literature is larger in the case of L-DIC; see for instance [ $50,28,40,48,21,49,47]$. It is worth noting that only few of these studies, $c f[48,21,49,47]$, pay attention to the interpolation which is required by DIC solving and which consequently affects the noise propagation. Nevertheless, the proposed formula from these publications is reduced to the L-DIC formulation. In [48], the prediction is only valid for subset translation. In [47] no comparison with experimental data is given. In [21, 49], validation is performed, but using an optical table, which does not reflect the environment of usual experimental mechanics laboratories.

A generalized predictive formula has recently been proposed in [4]. It is worth noting that this proposed formula is applicable to both L- and G-DIC. It was numerically validated in [4] using synthetic data, and compared with the usual formula available in the G-DIC literature [36]. The validity of this formula relies on various hypotheses, and the present paper aims to validate, using experimental data coming from a standard experimental mechanics laboratory, both these hypotheses and this predictive formula. In particular, because of the particular laboratory conditions, violations of some of these assumptions occur. They are highlighted here, and specific pre-processing tools are proposed to circumvent the adverse experimental conditions. The predictive formula is finally validated after applying these tools.

This paper is organized as follows. Section 2 recalls the basics of DIC; more specifically, the displacement resolution predictive formula is carefully elaborated. In Section 3, this prediction of the resolution is compared with its experimental counterparts deduced from measurements. It highlights the violation of the assumptions that have been introduced to obtain the predictive formula. Section 4 is dedicated to the presentation and implementation of tools that ensure the assumptions to be satisfied, and the predictive formula to be validated. Section 5 concludes the present study.

\section{Digital Image Correlation and displacement resolution prediction}

This section briefly recalls the basics of DIC, introduces the notation and provides the formula for the prediction of displacement resolution introduced in [4].

The DIC framework employed here mainly relies on G-DIC. Recent studies aim at comparing Local and Global versions of DIC [51, 26, 18], which is not the scope of the present paper. Note that L-DIC can be considered as a particular case of multiple independent G-DIC. The nomenclature in Tab.(1) summarizes the notation used in the paper. Let us note $(f)$ the image of the zone of interest on the specimen in the reference state, and $(g)$ the image of this zone in the deformed state. Let $\underline{O}$ be the top left corner of the sensor and $\left(\underline{e}_{1}, \underline{e}_{2}\right)$ two unit vectors attached to the pixel coordinates. $f\left(\underline{x}_{\mathrm{p}}\right)$ and $g\left(\underline{x}_{\mathrm{p}}\right)$ hence denote the pixel intensity (gray level) at each pixel position $\underline{x}_{\mathrm{p}}$ expressed in the basis $\left(\underline{O} ; \underline{e}_{1}, \underline{e}_{2}\right)$. Assuming that the pixel intensities are simply translated from $(f)$ to $(g)$, we obtain:

$$
f\left(\underline{x}_{\mathrm{p}}\right) \approx g\left(\underline{x}_{\mathrm{p}}+\underline{U}\left(\underline{x}_{\mathrm{p}}\right)\right)
$$

where $\underline{U}$ is the displacement of the specimen material points observed in the sensor coordinate system. 


\begin{tabular}{|c|c|}
\hline$\left(\underline{O} ; \underline{e}_{1}, \underline{e}_{2}\right)$ & Camera sensor coordinate system \\
\hline RoI & Region of Interest \\
\hline$N_{p}$ & Number of pixels in the RoI \\
\hline$\underline{\underline{x_{p}}}$ & Arbitrary pixel location \\
\hline$(f)$ & Reference speckle image \\
\hline$(g)$ & Current speckle image \\
\hline$\underline{u}$ & DIC output displacement using $(f) \&(g)$ \\
\hline $\bar{L}$ & Projection of DIC displacement shape function on the reference image gradient, see Eq.(5) \\
\hline$M$ & DIC tangent operator, see Eq.(5) \\
\hline$P$ & Interpolation operator, once the DIC scheme has converged, see Eq.(6) \\
\hline$n_{h}$ & Noise corrupting the image $(h)$ \\
\hline$(\tilde{h})$ & Image $(h)$ corrupted by acquisition noise, i.e., $\tilde{h}=h+n_{h}$ \\
\hline$(\mathrm{f})$ & Reference grid image \\
\hline (g) & Current grid image \\
\hline$\underline{\underline{\mathrm{u}}}$ & DIC output displacement using (f) \& (g) \\
\hline$\langle h\rangle$ & Pixel mean value of images $\left(h_{t}\right)_{1 \leq N_{\text {Img }}}$ \\
\hline$\sigma_{h, \mathrm{p}}$ & Pixel standard deviation of images $\left(h_{t}\right)_{1 \leq N_{\mathrm{img}}}$ \\
\hline$\sigma_{h}$ & Image standard deviation of images $\left(h_{t}\right)_{1 \leq N_{\mathrm{img}}}$ \\
\hline$\left\langle\overline{\left\langle u_{l}\right\rangle}\right.$ & Observed mean value of the $\ell^{\text {th }}$-component displacement \\
\hline$\sigma_{u_{\ell}^{o}}$ & Observed standard deviation of the $\ell^{\text {th }}$-component displacement \\
\hline$\sigma_{u_{\ell}^{p}}$ & Predicted standard deviation of the $\ell^{\text {th }}$-component displacement \\
\hline $\bar{\sigma}_{u_{\ell}^{p}}^{p}$ & Predicted standard deviation of the $\ell^{\text {th }}$-component displacement, with the relaxed hypothesis $\overline{\mathcal{H}}_{1}$ \\
\hline$\rho$ & Ratio between observed resolution of the displacement vertical component $\sigma_{u_{2}^{o}}$ and predicted resolution $\sigma_{u_{2}^{p}}$ \\
\hline $\bar{\rho}$ & Ratio between observed resolution of the displacement vertical component $\sigma_{u_{2}^{o}}$ and predicted resolution $\bar{\sigma}_{u_{2}^{p}}$ \\
\hline$\overline{\text { GAT }}$ & Generalized Anscombe Transform, see Section 3.3 \\
\hline$\left(h_{\mathrm{G}}\right)$ & Image $(h)$ after GAT \\
\hline$\underline{\underline{u}}_{\mathrm{G}}$ & DIC output displacement using $\left(f_{\mathrm{G}}\right) \&\left(g_{\mathrm{G}}\right)$ \\
\hline NRSR & Non-Random Signal Reduction, see Section 3.4 \\
\hline$\left(h_{\mathrm{N}}\right)$ & Image $(h)$ after NRSR \\
\hline$\underline{u}_{\mathrm{N}}$ & DIC output displacement using $\left(f_{\mathrm{N}}\right) \&\left(g_{\mathrm{N}}\right)$ \\
\hline$\left(h_{\mathrm{N}-\mathrm{G}}\right)$ & Image $(h)$ after NRSR \& GAT \\
\hline$\underline{\underline{u}}_{\mathrm{N}-\mathrm{G}}$ & DIC output displacement using $\left(f_{\mathrm{N}-\mathrm{G}}\right) \&\left(g_{\mathrm{N}-\mathrm{G}}\right)$ \\
\hline$a$ & Scalar, $a \in \Re$ \\
\hline$\underline{a}$ & First-rank tensor, here meaning $\underline{a} \in \Re^{2}$ or more specifically, $\exists\left(a_{1}, a_{2}\right) \in \Re^{2} \mid \underline{a}=a_{1} \underline{e}_{1}+a_{2} \underline{e}_{2}$ \\
\hline$a$ & Data vector of size $N_{p}$ or $N_{\text {DoF }} ;$ its i ${ }^{\text {th }}$ term is denoted $[\boldsymbol{a}]_{i}$ and $[\boldsymbol{a}]_{i} \in \Re$ \\
\hline$A$ & Matrix; the term in its $\mathrm{i}^{\text {th }}$ line and $\mathrm{j}^{\text {th }}$ column is denoted $[\boldsymbol{A}]_{i j}$ and $[\boldsymbol{A}]_{i j} \in \Re$ \\
\hline
\end{tabular}

\section{Table 1: Notation}

Within DIC, an approximated displacement $\underline{u}$ is usually introduced using a set of $N_{\text {DoF }}$

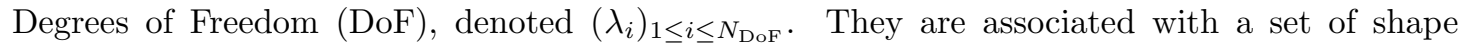
functions, $\left(\underline{\varphi}_{i}\left(\underline{x}_{\mathrm{p}}\right)\right)_{1 \leq i \leq N_{\mathrm{DoF}}}$. Thus, $\underline{u}$ is defined as follows

$$
\underline{u}\left(\underline{x}_{\mathrm{p}}, \boldsymbol{\lambda}\right)=\sum_{i=1}^{N_{\text {DoF }}} \lambda_{i} \underline{\varphi}_{i}\left(\underline{x}_{\mathrm{p}}\right)
$$

where $\boldsymbol{\lambda}$ is the vector composed of the $\operatorname{DoF}\left(\lambda_{i}\right)_{1 \leq i \leq N_{\text {DoF }}}$. 
DIC attempts to find the best approximated displacement $\underline{u}^{\text {opti }}$, i.e., the displacement satisfying Eq.(2), which minimizes the sum of the squares of the optical residual over a given Region of Interest (RoI):

$$
\underline{u}^{\text {opti }}\left(\underline{x}_{\mathrm{p}}\right)=\underline{u}\left(\underline{x}_{\mathrm{p}}, \boldsymbol{\lambda}^{\text {opti }}\right) \text { with } \boldsymbol{\lambda}^{\text {opti }}=\underset{\boldsymbol{\lambda}}{\operatorname{Argmin}}\left\{\sum_{\underline{x}_{\mathrm{p}} \in \operatorname{RoI}}\left\{f\left(\underline{x}_{\mathrm{p}}\right)-g\left(\underline{x}_{\mathrm{p}}+\underline{u}\left(\underline{x}_{\mathrm{p}}, \boldsymbol{\lambda}\right)\right)\right\}^{2}\right\} .
$$

We choose here a Sum of Square Difference (SSD) criterion to drive the DIC minimization. Of course another criterion could be chosen, such as the Zero-mean Normalized Sum of Square Difference (ZNSSD), for instance. This choice impacts the solution, and the associated resolution. A detailed study is proposed in [27]. Nevertheless, the problem stated here can also be elaborated for any other criterion and the associated predictive formula can be developed.

As detailed in [25] and briefly recalled in Appendix (A), this minimization problem is iteratively solved by updating $\boldsymbol{\lambda}$ with a modified Gauss-Newton scheme:

$$
\lambda^{\mathrm{it}+1}=\lambda^{\mathrm{it}}+\Delta \lambda^{\mathrm{it}}
$$

where "it" is the iteration count. The correction between two iterations is such that:

$$
\boldsymbol{\Delta} \boldsymbol{\lambda}^{\mathrm{it}}=\boldsymbol{M}^{-1} \boldsymbol{b} \text { with }\left\{\begin{array} { l } 
{ \boldsymbol { M } = \boldsymbol { L } \boldsymbol { L } ^ { T } } \\
{ \boldsymbol { b } = \boldsymbol { L } \boldsymbol { r } ( \boldsymbol { \lambda } ^ { \mathrm { it } } ) }
\end{array} \text { and } \left\{\begin{array}{l}
{[\boldsymbol{L}]_{i j}=\nabla f\left(\underline{x}_{\mathrm{j}}\right) \cdot \underline{\varphi}_{i}\left(\underline{x}_{\mathrm{j}}\right)} \\
{\left[\boldsymbol{r}\left(\boldsymbol{\lambda}^{\mathrm{it}}\right)\right]_{i}=f\left(\underline{x}_{\mathrm{i}}\right)-g\left(\underline{x}_{\mathrm{i}}+\underline{u}\left(\underline{x}_{\mathrm{i}}, \boldsymbol{\lambda}^{\mathrm{it}}\right)\right)}
\end{array}\right.\right.
$$

where "." denotes the standard Euclidean product. The DoF are updated until a convergence criterion is satisfied. Their optimized values are gathered in $\lambda^{\text {opti }}$. We choose here the usual relative threshold $\|\boldsymbol{b}\| \leq 10^{-5} \times\left\|\boldsymbol{b}^{0}\right\|$, with $\left\|\boldsymbol{b}^{0}\right\|$ defined as the initial value of $\|\boldsymbol{b}\|$, as in [5].

Since image $(g)$ is known at the pixel positions, an interpolation is required for the calculation

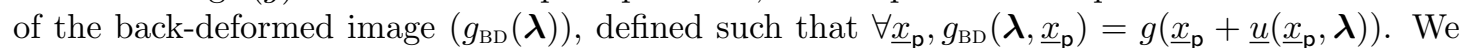
can introduce an operator $\boldsymbol{P}(\boldsymbol{\lambda})$, which interpolates $\boldsymbol{g}$. With vector notation, $[\boldsymbol{g}]_{i}=g\left(\underline{x}_{\mathrm{i}}\right)$ and $\left[\boldsymbol{g}_{\mathrm{BD}}(\boldsymbol{\lambda})\right]_{i}=g_{\mathrm{BD}}\left(\boldsymbol{\lambda}, \underline{x}_{\mathrm{i}}\right)$. We have:

$\boldsymbol{g}_{\mathrm{BD}}(\boldsymbol{\lambda})=\boldsymbol{P}(\boldsymbol{\lambda}) \boldsymbol{g}$, with $\left\{\begin{array}{l}{[\boldsymbol{P}(\boldsymbol{\lambda})]_{i j}=\omega_{i j} \text { if } \underline{x}_{\mathrm{j}} \in E_{i} \text { and } 0 \text { otherwise }} \\ E_{i} \text { denotes the subset of pixels involved in the interpolation of } \underline{x}_{\mathrm{p}}+\underline{u}\left(\underline{x}_{\mathrm{p}}, \boldsymbol{\lambda}\right) . \\ \omega_{i j} \text { corresponds to the weight associated with each pixel of } E_{i}\end{array}\right.$.

It is worth noting that this operator can be employed to represent either bilinear or bicubic interpolations. In this study, the bilinear interpolation has been implemented. Once the algorithm has converged, $\boldsymbol{P}$ simply refers to $\boldsymbol{P}\left(\boldsymbol{\lambda}^{\text {opti }}\right)$. It should be noted that, if $\underline{x}_{\mathrm{p}}+\underline{u}_{\mathrm{x}}\left(\underline{x}_{\mathrm{p}}, \boldsymbol{\lambda}\right)$ lies on the discrete pixel grid, no interpolation is needed; in this case, for any $i, E_{i}$ reduces to a single pixel and $\omega_{i, j}$ equals 0 except for a single index $j$.

When DIC is applied to experimental data, it turns out that the images are impaired by sensor noise, which propagates to displacement maps through the Gauss-Newton scheme. Denoting the sensor noise at each pixel $\underline{x}_{\mathrm{p}}$ by a function $n\left(\underline{x}_{\mathrm{p}}\right)$, the actual intensities of both images are modeled by $\widetilde{f}\left(\underline{x}_{\mathrm{p}}\right)=f\left(\underline{x}_{\mathrm{p}}\right)+n_{f}\left(\underline{x}_{\mathrm{p}}\right)$ and $\widetilde{g}\left(\underline{x}_{\mathrm{p}}\right)=g\left(\underline{x}_{\mathrm{p}}\right)+n_{g}\left(\underline{x}_{\mathrm{p}}\right)$. This noise directly impacts the resolution of measurement $\boldsymbol{\lambda}$, hence of the displacement. Assuming that both $n_{f}\left(\underline{x}_{\mathrm{p}}\right)$ and $n_{g}\left(\underline{x}_{\mathrm{p}}\right)$ are $0-$ mean Gaussian white noise (independent Gaussian variables of equal standard deviation $\sigma_{f}$ assumed to be constant across the images), the authors of [2] quantify a global indicator of the expected resolution over the whole displacement maps. Réthoré, in [35], explicitly elaborated the perturbation $\boldsymbol{\delta} \boldsymbol{\lambda}$ of the optimal values of the DoF due to acquisition noise, with the same assumption of (spatially constant) Gaussian white noise. Finally, recent advances introduced in 
[4] enable a finer calculation of the predictive values of the resolution when sub-pixel displacement occurs. Let us briefly recall here the different steps leading to the result obtained in [4], in order to highlight the four assumptions $\mathcal{H}_{1}, \mathcal{H}_{2}, \mathcal{H}_{3}$ and $\mathcal{H}_{4}$ that are made.

- $\left(\mathcal{H}_{1}\right)$ : Sensor noises $n_{f}(\underline{x})$ and $n_{g}(\underline{x})$ are assumed to be 0-mean Gaussian white noises of standard deviation $\sigma_{f}$. $\sigma_{f}$ is thus assumed to be constant throughout images $\tilde{f}$ and $\tilde{g}$.

- $\left(\mathcal{H}_{2}\right)$ : Perturbation $\boldsymbol{\delta} \boldsymbol{\lambda}$ is assumed to be small enough with respect to optimized DoF values $\boldsymbol{\lambda}^{\text {opti }}$ themselves to allow the first-order Taylor approximation introduced within the modified Gauss-Newton scheme of the DIC solver. This leads to

$$
\boldsymbol{\delta} \boldsymbol{\lambda}=\tilde{\boldsymbol{M}}^{-1} \tilde{\boldsymbol{b}}
$$

where $\tilde{\boldsymbol{M}}$ and $\tilde{\boldsymbol{b}}$ are defined as in Eq.(5), by replacing $f$ and $g$ by the noisy $\tilde{f}$ and $\tilde{g}$ respectively.

- $\left(\mathcal{H}_{3}\right)$ : The image gradients of $\tilde{f}$ and $\tilde{g}$ are approximated by the one of the noiseless images:

$$
\underline{\nabla}(\tilde{f}) \sim \underline{\nabla}(f) \text { and } \underline{\nabla}(\tilde{g}) \sim \underline{\nabla}(g) .
$$

- $\left(\mathcal{H}_{4}\right)$ : The noise is supposed to have a small effect on the DIC solution itself. Thus, we assume that the fluctuation does not affect the interpolation operator $\boldsymbol{P}$. Moreover, we deduce from Eq.(5)

$$
\boldsymbol{L} \boldsymbol{r}\left(\boldsymbol{\lambda}^{\mathrm{opti}}\right) \sim 0
$$

Remark 2.1 In practice, only the gradient of the reference image is required during DIC solving, as expressed above in Eq.(5). To ensure the satisfaction of hypothesis $\mathcal{H}_{3}$, the gradient of the reference noiseless image $\underline{\nabla}(f)$ is built on a specific image $\langle f\rangle$ obtained by averaging several images that capture the reference state. The DIC tangent operator is defined through $\underline{\nabla}\langle f\rangle$, thus using a large number of images. It is thus considered to be noiseless. Moreover, such a definition of the DIC tangent operator enables noise propagation through the DIC solving process to be alleviated. Indeed, taking noise into account in the tangent operator leads to a complex numerical calculation. An example in another context can be found in [44].

Thanks to hypotheses $\mathcal{H}_{3}$ and $\mathcal{H}_{4}, \tilde{\boldsymbol{M}}$ and $\tilde{\boldsymbol{b}}$ become, at convergence

$$
\left\{\begin{array}{l}
\tilde{\boldsymbol{M}}=\boldsymbol{M} \\
\tilde{\boldsymbol{b}}=\boldsymbol{L}(\boldsymbol{f}-\boldsymbol{P g})+\boldsymbol{L}\left(\boldsymbol{n}_{f}-\boldsymbol{P} \boldsymbol{n}_{g}\right)
\end{array} \text { with } \forall i,\left[\boldsymbol{n}_{f}\right]_{i}=n_{f}\left(\underline{x}_{\mathrm{i}}\right) \text { and }\left[\boldsymbol{n}_{g}\right]_{i}=n_{g}\left(\underline{x}_{\mathrm{i}}\right) .\right.
$$

Thanks to hypothesis $\mathcal{H}_{1}, \boldsymbol{n}_{f}$ and $\boldsymbol{n}_{g}$ are 0 -mean Gaussian random vectors. Moreover, the following proposition enables us to propagate the noise through linear mapping:

Proposition 2.1 If $\boldsymbol{x}$ is a Gaussian random vector of size $k$, mean $\langle\boldsymbol{x}\rangle$ and covariance matrix $\boldsymbol{\Sigma}_{\boldsymbol{x}}$, and if $\boldsymbol{A}$ is a matrix of size $l \times k$, then $\boldsymbol{y}=\boldsymbol{A} \boldsymbol{x}$ is a Gaussian random vector of mean $\langle\boldsymbol{y}\rangle=\boldsymbol{A}\langle\boldsymbol{x}\rangle$, and covariance matrix $\boldsymbol{\Sigma}_{\boldsymbol{y}}=\boldsymbol{A} \boldsymbol{\Sigma}_{\boldsymbol{x}} \boldsymbol{A}^{T}$ (for further details, see [15] for instance).

$\tilde{\boldsymbol{b}}$ is thus a Gaussian vector of mean $\boldsymbol{L}(\boldsymbol{f}-\boldsymbol{P g})$ and covariance matrix $\boldsymbol{L}\left(\sigma_{f}^{2} \boldsymbol{I}+\boldsymbol{P} \sigma_{f}^{2} \boldsymbol{I} \boldsymbol{P}^{T}\right) \boldsymbol{L}^{T}=$ $\sigma_{f}^{2} \boldsymbol{L}\left(\boldsymbol{I}+\boldsymbol{P} \boldsymbol{P}^{T}\right) \boldsymbol{L}^{T}, \boldsymbol{I}$ being the identity matrix.

By composition, $\boldsymbol{\delta} \boldsymbol{\lambda}=\boldsymbol{M}^{-1} \tilde{\boldsymbol{b}}$ is a Gaussian vector of mean $\boldsymbol{M}^{-1} \boldsymbol{L}(\boldsymbol{f}-\boldsymbol{P g})$. Thanks to hypothesis $\mathcal{H}_{4}$, this bias only corresponds to the one introduced by the interpolation scheme [40]. An interested reader can refer to [48], where $\mathcal{H}_{4}$ is relaxed, to analyze the effect of the sensor noise on the bias. It is worth noting that the resulting procedure leads to high order 
terms in the covariance elaboration. Since we focus here on the resolution, i.e., on the DoF covariance $\boldsymbol{\Sigma}_{\boldsymbol{\delta} \boldsymbol{\lambda}}$, we still assume $\mathcal{H}_{4}$ valid.

Moreover, since $\boldsymbol{M}$ (hence $\boldsymbol{M}^{-1}$ ) is symmetric, the covariance matrix of $\boldsymbol{\delta} \boldsymbol{\lambda}$ satisfies

$$
\begin{gathered}
\boldsymbol{\Sigma}_{\boldsymbol{\delta} \boldsymbol{\lambda}}=\boldsymbol{M}^{-1}\left(\sigma_{f}^{2} \boldsymbol{L}\left(\boldsymbol{I}+\boldsymbol{P} \boldsymbol{P}^{T}\right) \boldsymbol{L}^{T}\right)\left(\boldsymbol{M}^{-1}\right)^{T}=\sigma_{f}^{2}\left(\boldsymbol{M}^{-1}(\underbrace{\boldsymbol{L} \boldsymbol{L}^{T}}_{\boldsymbol{M}}) \boldsymbol{M}^{-1}+\boldsymbol{M}^{-1} \boldsymbol{L} \boldsymbol{P} \boldsymbol{P}^{T} \boldsymbol{L}^{T} \boldsymbol{M}^{-1}\right) \\
=\sigma_{f}^{2}\left(\boldsymbol{M}^{-1}+\boldsymbol{M}^{-1} \boldsymbol{L} \boldsymbol{P} \boldsymbol{P}^{T} \boldsymbol{L}^{T} \boldsymbol{M}^{-1}\right) .
\end{gathered}
$$

In the particular case of Finite Element-based-DIC (introduced in [2], compared with usual LDIC in $[18]$ and recently used in $[12,31]$ for instance), $\boldsymbol{\lambda}$ corresponds to the vector of the nodal displacements. At each node $k$ of coordinate $\underline{x}_{k}$, there is a pair $\left(k_{1}, k_{2}\right)$ such that

$$
\underline{u}\left(\underline{x}_{k}\right)=\lambda_{k_{1}} \underline{e}_{1}+\lambda_{k_{2}} \underline{e}_{2} .
$$

The standard deviations $\sigma_{u_{1}}^{p}$ (resp. $\sigma_{u_{2}}^{p}$ ) of the first (resp. second) component of the displacement of node $k$ can thus be computed, since this is the square root of the diagonal terms of the covariance matrix Eq.(11) of $\boldsymbol{\delta} \boldsymbol{\lambda}$. This leads to:

$$
\sigma_{u_{\ell}\left(\underline{x}_{k}\right)}^{p}=\sqrt{\left[\boldsymbol{M}^{-1}+\boldsymbol{M}^{-1} \boldsymbol{L} \boldsymbol{P} \boldsymbol{P}^{T} \boldsymbol{L}^{T} \boldsymbol{M}^{-1}\right]_{l l}} \times \sigma_{f}, \quad \ell \in\{1,2\} \text { and } l=\left\{\begin{array}{l}
k_{1} \text { if } \ell=1 \\
k_{2} \text { otherwise }
\end{array} .\right.
$$

Note that if the sub-pixel part of the displacement is null, $\boldsymbol{P} \boldsymbol{P}^{T}$ is equal to the identity matrix, and thus Eq.(13) reduces exactly to that given in [35], which does not take into account the sub-pixel displacement.

It is worth noting that hypothesis $\mathcal{H}_{1}$ can be relaxed in $\overline{\mathcal{H}}_{1}$ by assuming $\left(n_{f}\right)$ and $\left(n_{g}\right)$ as 0-mean Gaussian noises of standard deviation $\sigma_{f}\left(\underline{x}_{\mathrm{p}}\right)$ which spatially vary. $\boldsymbol{n}_{f}$ and $\boldsymbol{n}_{g}$ would be, by consequence, $0-$ mean Gaussian random vectors of covariance matrices $\boldsymbol{\Sigma}_{\boldsymbol{n}_{f}}$ and $\boldsymbol{\Sigma}_{\boldsymbol{n}_{g}}$. Both matrices are diagonal, such that for any $i,\left[\boldsymbol{\Sigma}_{\boldsymbol{n}_{f}}\right]_{i i}=\sigma_{f}\left(\underline{x}_{\mathrm{i}}\right)^{2}$ and $\left[\boldsymbol{\Sigma}_{\boldsymbol{n}_{g}}\right]_{i i}=\sigma_{g}\left(\underline{x}_{\mathrm{i}}\right)^{2}$. This case would lead to a covariance matrix of $\delta \boldsymbol{\lambda}$ that satisfies

$$
\overline{\boldsymbol{\Sigma}}_{\boldsymbol{\delta} \boldsymbol{\lambda}}=\boldsymbol{M}^{-1}\left(\boldsymbol{L}\left(\boldsymbol{\Sigma}_{\boldsymbol{n}_{f}}+\boldsymbol{P} \boldsymbol{\Sigma}_{\boldsymbol{n}_{g}} \boldsymbol{P}^{T}\right) \boldsymbol{L}^{T}\right) \boldsymbol{M}^{-1}
$$

The corresponding standard deviations $\sigma_{u_{1}}^{p}$ (resp. $\sigma_{u_{2}}^{p}$ ) of the first (resp. second) component of the displacement of node $k$ is

$$
\bar{\sigma}_{u_{\ell}\left(\underline{x}_{k}\right)}^{p}=\sqrt{\left[\boldsymbol{M}^{-1}\left(\boldsymbol{L}\left(\boldsymbol{\Sigma}_{\boldsymbol{n}_{f}}+\boldsymbol{P} \boldsymbol{\Sigma}_{\boldsymbol{n}_{g}} \boldsymbol{P}^{T}\right) \boldsymbol{L}^{T}\right) \boldsymbol{M}^{-1}\right]_{l l}}, \quad \ell \in\{1,2\} \text { and } l=\left\{\begin{array}{l}
k_{1} \text { if } \ell=1 \\
k_{2} \text { otherwise }
\end{array} .\right.
$$

Presented in [4], a specific study aims at thoroughly introducing this generalized prediction of displacement resolution, validating it with numerical data and discussing its benefits with respect to the usual formula given in [35]. To the best of the authors' knowledge, despite the fact that predicted values of the displacement resolution, even in its raw form from [35], have already been used to highlight the confidence intervals of the results $(c f[2,12])$ or to determine the effect of acquisition noise on the post-processing of the DIC output $(c f[34,12,9])$, there is no study focused on the thorough validation of this predictive formula. The two next sections validate it in a controlled setting.

\section{Experimental validation: vertical translation}

This section is dedicated to the experimental validation of the predictive formula given in Eq.(13). The determination of the observed resolution is introduced here. Section 3.1 is dedicated to the 
presentation of (i) the experimental set-up and associated methods, (ii) the DIC implementation used for displacement map measurement and (iii) the statistical analysis elaborated using the experimental data. Section 3.2 deals with these first estimations of the resolution and focuses on the non-verification of the predictive formula. In addition, violations of the hypotheses required by the formula elaboration are highlighted. Section 3.3 focuses on the actual sensor noise, which does not satisfy assumption $\mathcal{H}_{1}$. A usual model for sensor noise is presented and two strategies are proposed to take it into account:

- $\left(\mathcal{S}_{1}\right)$ introducing a tool, namely the Generalized Anscombe Transform (GAT), that stabilizes the variance of any Poisson-Gaussian process to ensure that hypothesis $\mathcal{H}_{1}$ is valid;

- $\left(\mathcal{S}_{2}\right)$ updating the image noise assumption required for the predictive formula elaboration, in other words, relaxing $\mathcal{H}_{1}$ to $\overline{\mathcal{H}}_{1}$ as introduced above.

The application of these strategies is then illustrated and discussed. It is however observed that Rigid Body Motion (RBM) also occurs during image acquisition, affecting the images and thus impairing the estimation of camera sensor noise properties. A second pre-process, NonRandom Signal Reduction (NRSR), is therefore presented in the last section; this softens the RBM effect and thus enables us to apply the sensor noise model. Predictive values given in Eq.(15) for $\mathcal{S}_{1}$ or in Eq.(13) for $\mathcal{S}_{2}$ and observed measurements of the resolution are compared. The results obtained confirm the validity of the predictive formula thanks to these two particular pre-processing strategies.

\subsection{Experimental set-up and methods}

A speckle pattern was painted on a rectangular plate specimen, as illustrated in Fig.(1a). This pattern was imaged using a SENSICAM QE camera (PCO, Kelheim, Germany) featuring a 12-bit/1376x1040-pixel cooled sensor and equipped with a 105-mm SIGMA lens (SIGMA) such that the Region of Interest $(\mathrm{RoI})$ had a dimension of $50 \times 34[\mathrm{~mm}]^{2}$, discretized into $1249 \times 846$ [pixel] ${ }^{2}$, see Fig.(1b-c). The RoI contains thus $N_{p}=1056654$ [pixel].

During each test, the speckle was illuminated by one led light source. This specific lighting system uses a continuous power source to ensure the absence of illumination flickering. The location of the light spot was deliberately adjusted in order to provide spatially non-uniform lighting, highlighting the possible effect of its heterogeneity. However, the spatial fluctuation of the illumination was smooth enough to maintain the constant brightness hypothesis (cf Eq.(1)). Moreover, the displacement was along the vertical direction, whereas the lighting gradient was mainly along the horizontal one.

The specimen was clamped only in the mobile (upper) grip of a tensile testing machine. In order to determine precisely the acquisition noise corrupting the images, 100 images were acquired at each stage of the test. The reference state was thus captured by a set of $N_{\mathrm{img}}$ images, i.e., images $\left(f_{t}\right)_{1 \leq t \leq N_{\mathrm{img}}}$, while the current state, defined after a $100[\mu \mathrm{m}]$ translation of the upper grip, was also captured through $N_{\text {img }}$ images, i.e., images $\left(g_{t}\right)_{1 \leq t \leq N_{\text {img }}}$, with $N_{\text {img }}=100$. With a pixel size equal to $40[\mu \mathrm{m}]$, the translation of $100[\mu \mathrm{m}](=2.5$ [pixel] $)$ was deliberately chosen in order to emphasize the consequence of DIC interpolation in its worst case. The available stack of images makes it possible to calculate empirical estimates of the mean value and standard deviation of the gray-level intensity at any pixel, and to observe various phenomena such as the influence of the gray level intensity on local noise in the displacement field.

The main characteristics of the statistical analysis, i.e., the pixel mean value $\langle f\rangle$, the pixel 


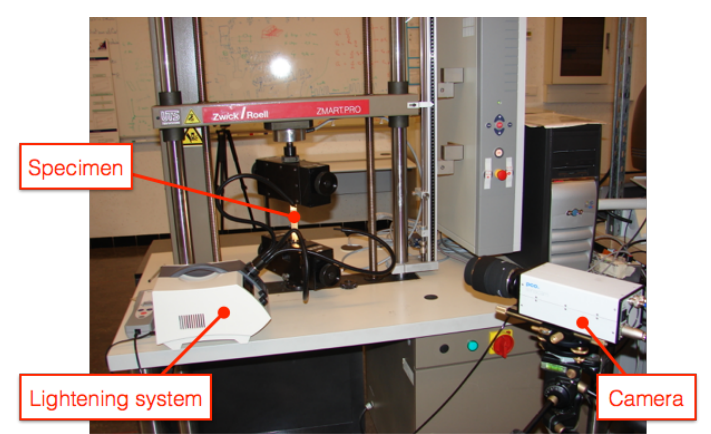

(a) Illustration of the set-up with its instrumentation (camera and lighting system)

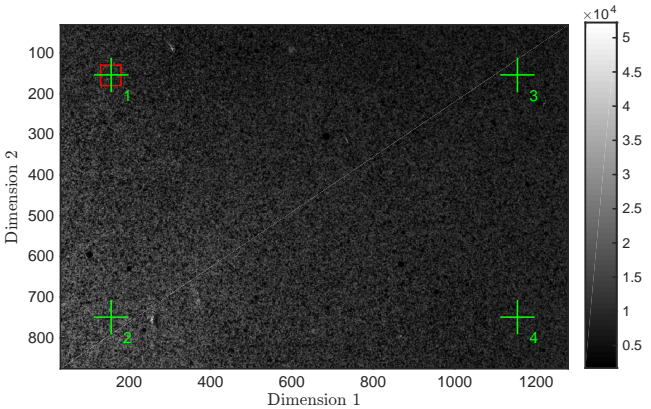

(b) Region of Interest [gray level]

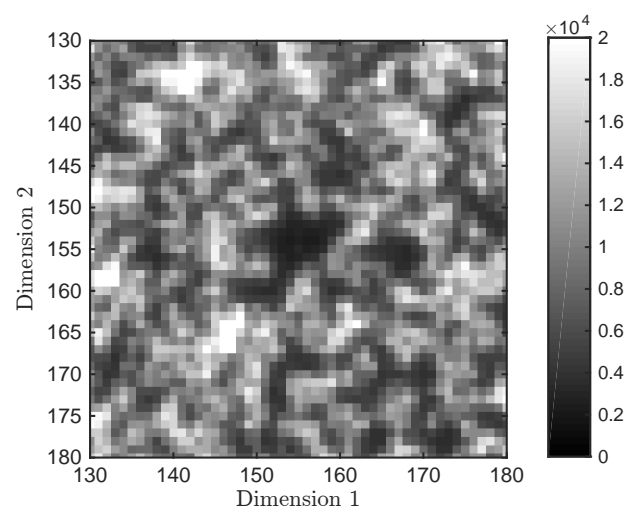

(c) Close-up of the RoI (red box in (b)) [gray level]

Figure 1: Illustration of the set-up, with the specimen and the speckle pattern. (a) Specimen, clamped only in the mobile (upper) grip of the tensile testing machine. The camera and the lighting system are visible. (b) RoI, the red box specifies the location of the close-up view and the crosses indicate the position of points $1-4$. (c) Close-up of one spot of the RoI, illustrating the random nature of the speckle and its high contrast. 


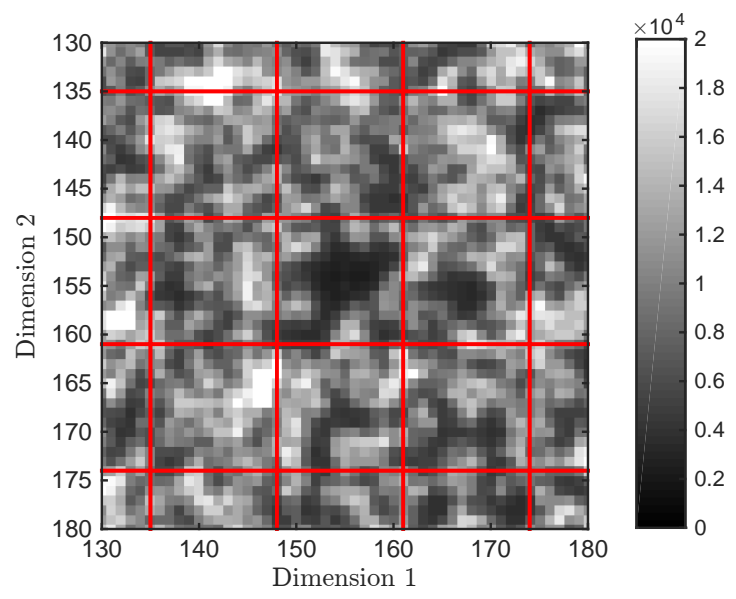

Figure 2: Detail of the speckle with the DIC mesh (same detail location as in Fig.1c).

standard deviation $\sigma_{f, \mathrm{p}}$ and the average image noise standard deviation $\sigma_{f}$ are defined as follows:

$$
\begin{aligned}
\langle f\rangle & =\frac{1}{N_{\mathrm{img}}} \sum_{t=1}^{N_{\mathrm{img}}} f_{t} ; \\
\sigma_{f, \mathrm{p}} & =\sqrt{\frac{1}{N_{\mathrm{img}}-1} \sum_{t=1}^{N_{\mathrm{img}}}\left(f_{t}-\langle f\rangle\right)^{2}} ; \\
\sigma_{f} & =\sqrt{\frac{1}{N_{p}} \sum_{\underline{x}_{\mathrm{p}} \in R o I} \sigma_{f, \mathrm{p}}^{2}}
\end{aligned}
$$

The subscript notation "p" is introduced to highlight the difference between the map $\sigma_{f, \mathrm{p}}$ (gray level standard deviation, evaluated at each pixel) and the scalar value $\sigma_{f}$ (image-wise gray level standard deviation).

For each pair of images $\left(f_{t}, g_{t}\right)_{1 \leq t \leq N_{\text {img }}}$, a G-DIC is performed in order to retrieve the prescribed translation which was applied to the specimen. A FE-based displacement field space is implemented, defined by $13 \times 13$ [pixel] $]^{2}$ bilinear square elements. Composed of $96 \times 65$ elements, the mesh contains 6402 nodes. This choice of element size was arbitrarily chosen. It is worth noting that similar studies with larger element sizes $(25 \times 25$ and $50 \times 50)$ were carried out and led to similar results. The displacement is thus defined through 12804 DoF. Fig.(2) illustrates this mesh at the top of the speckle for the close-up region introduced in Fig.(1b).

Using the $N_{\mathrm{img}}$ DIC realizations, an empirical displacement resolution, i.e., standard deviation $\sigma_{u_{\ell}}^{e}$, is computed, $\ell=\{1,2\}$ referring to the component direction. $\sigma_{u_{\ell}}^{e}$ is defined at each 
pixel by:

$$
\begin{aligned}
& \sigma_{u_{\ell}}^{e}=\sqrt{\frac{1}{N_{\mathrm{img}}-1} \sum_{t=1}^{N_{\mathrm{img}}}\left(u_{\ell t}-\left\langle u_{\ell}\right\rangle\right)^{2}} \\
& \text { with }\left\langle u_{\ell}\right\rangle=\frac{1}{N_{\mathrm{img}}} \sum_{t=1}^{N_{\mathrm{img}}} u_{\ell t} .
\end{aligned}
$$

Finally, a ratio denoted $\rho$ is introduced. It is defined as the ratio between the empirical standard deviation of the vertical displacement field $\sigma_{u_{2}}^{e}$, and the predicted one $\sigma_{u_{2}}^{p}$ of Eq.(13), i.e., $\rho=\frac{\sigma_{u_{2}}^{e}}{\sigma_{u_{2}}^{p}}$. It is evaluated at each nodal coordinate. To avoid any extra interpolation, nodal coordinates are defined at pixel locations.

\subsection{First comparison of predicted values with empirical measurements of displacement resolution maps}

Fig.(3) illustrates the empirical standard deviations of the displacement $\sigma_{u_{2}}^{e}$, its predicted counter part $\sigma_{u_{2}}^{p}$ and their ratio $\rho$. Each of these quantities was evaluated at each nodal coordinate. We can notice the spatial dispersion of the ratio map, indicating that extrinsic parameters affecting the DIC algorithm have an effect on the displacement resolution, and have not been taken correctly into account in the predictive formula. While the observed resolution $\sigma_{u_{2}}^{e}$ distribution appears to be relatively flat, a gradient in the predicted one $\sigma_{u_{2}}^{p}$ can be observed. This gradient distribution seems to be correlated with the light intensity. Indeed, the brightest location (i.e., the bottom left corner, $c f$ Fig.(1b)) corresponds to the zone where the resolution is underestimated and vice versa. This point clearly highlights the over-conservative assumption made concerning the image noise definition $\sigma_{f}$, i.e., hypothesis $\mathcal{H}_{1}$. In $\mathcal{H}_{1}$, this noise is indeed assumed to be homoscedastic, which means that it is not related to the gray level intensity.

This assumption is however not satisfied, as illustrated in Fig.(4), which plots the standard deviation $\sigma_{f, \mathrm{p}}$ against the gray level of the mean value $\langle f\rangle$ at each pixel. A relationship between the two quantities is indeed obvious.

This feature of the camera sensor is already observable by comparing the pixel mean values $\langle f\rangle$ and the pixel standard deviation $\sigma_{f, \mathrm{p}}$, which are illustrated in Fig.(5). In the two close-ups shown in Fig. (5c-d), the same patterns are recognizable on both maps. The pixels which capture the highest quantity of light exhibit the highest standard deviation. In other words, the acquisition process of the camera sensor is not homoscedastic, but heteroscedastic. Its acquisition noise is correlated with the magnitude of the signal it captures. The next section of the paper deals with the noise heteroscedasticity of camera sensors and proposes two strategies to circumvent it.

\subsection{Incorporating the signal-dependent variance of sensor noise}

Hypothesis $\mathcal{H}_{1}$ requires the image noise to be 0-mean Gaussian white noise. However usual camera sensor noises are classically modeled with a heteroscedastic Poisson-Gaussian distribution, and hypothesis $\mathcal{H}_{1}$ cannot be satisfied anymore. Two strategies were investigated to tackle this issue: (i) already available in the image processing community, the Generalized Anscombe Transform (GAT) has been used here. It is a numerical method that enables us to correct the noise features of images into a homoscedastic process, and moreover into Gaussian noise of 0 -mean, which would then enables the satisfaction of $\mathcal{H}_{1}$; (ii) this hypothesis can also be relaxed into $\overline{\mathcal{H}}_{1}$ by assuming the image noise to be a 0 -mean Gaussian of standard deviation $\sigma_{f, \mathrm{p}}\left(\underline{x}_{\mathrm{p}}\right)$, which 


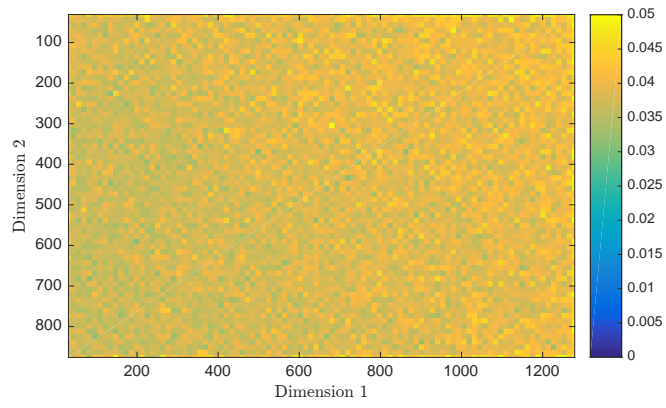

(a) Map of $\sigma_{u_{2}}^{e}$ [pixel]

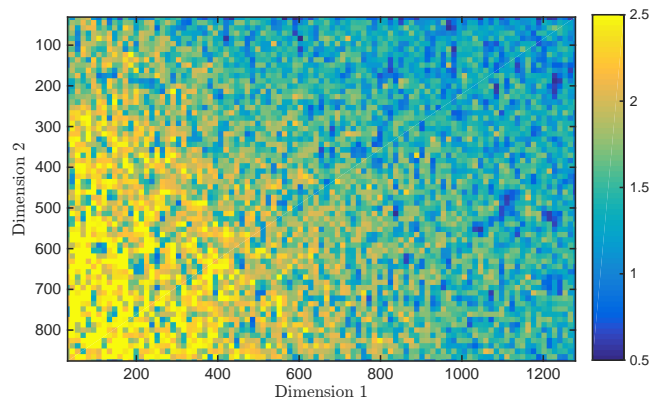

(c) Map of ratio $\rho[-]$

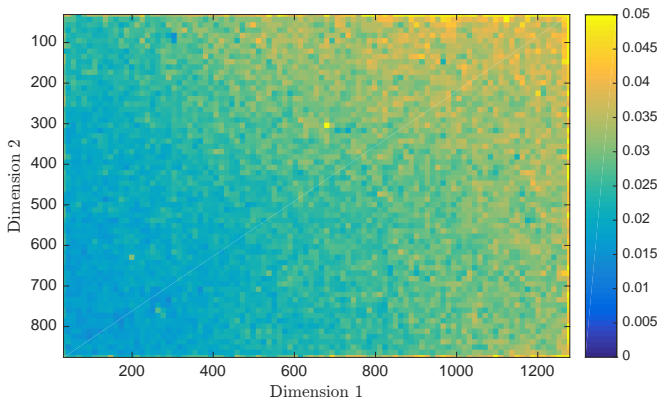

(b) Map of $\sigma_{u_{2}}^{p}$ [pixel]

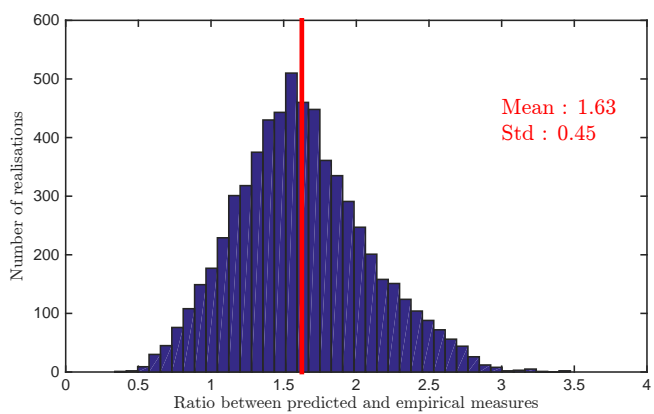

(d) Histogram of ratio $\rho[-]$

Figure 3: Empirical and predicted standard deviation of the vertical displacement, and their ratio $\rho$.

spatially changes. In this section, the standard sensor noise model is recalled, two proposed strategies to take it into account are presented, and the results obtained are discussed.

\subsubsection{Sensor noise model}

With standard exposure times, it is possible to neglect the dark signal of the CCD, in such a way that the raw output of a linear camera (i.e., the raw sensor output, without any image processing) is proportional to the quantity of light photons arriving at a CCD sensor cell, plus read-out noise and quantization noise. The following stochastic model (or with slight variations) is often used $[16,11]$

$$
f\left(\underline{x}_{\mathrm{p}}\right)=\mathcal{G}_{0} \times \eta_{\nu\left(\underline{x}_{\mathrm{p}}\right)}\left(\underline{x}_{\mathrm{p}}\right)+\eta_{f}\left(\underline{x}_{\mathrm{p}}\right)
$$

where:

- $f\left(\underline{x}_{\mathrm{p}}\right)$ is the intensity or the gray level measured at the photosite corresponding to pixel $\underline{x}_{\mathrm{p}}$ in the raw output image;

- $\mathcal{G}_{0}>0$ is the gain of the electronic system; 


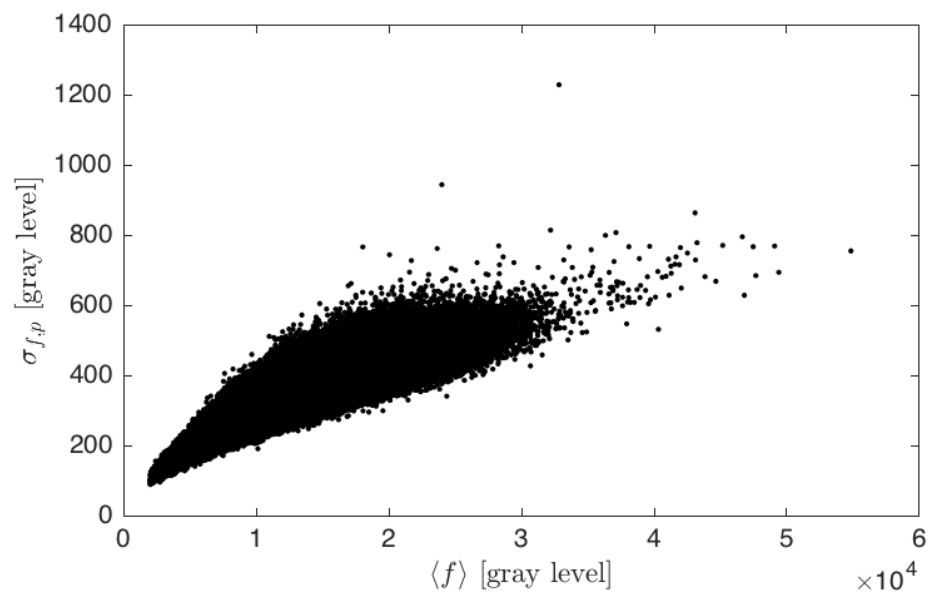

Figure 4: Map of the standard deviation $\left(\sigma_{f, \mathrm{p}}\right)$ against mean value $(\langle f\rangle)$, in gray level, at each pixel position.

- The number of generated electrons $\eta_{\nu\left(\underline{x}_{\mathrm{p}}\right)}$ at $\underline{x}_{\mathrm{p}}$ is a random variable following a Poisson distribution of mean value $\nu\left(\underline{x}_{\mathrm{p}}\right)$, which denotes the average number of electrons produced by a part of the incident photons (the proportion depends on the quantum efficiency);

- $\eta_{f}\left(\underline{x}_{\mathrm{p}}\right)$ is a Gaussian random vector of mean $\left\langle\eta_{f}\right\rangle$ and standard deviation $\sigma_{\eta_{f}}$ (caused by readout and quantization noise). $\left\langle\eta_{f}\right\rangle$ is a fixed offset value imposed by the sensor manufacturer;

- The random variables $\eta_{\nu\left(\underline{x}_{\mathrm{p}}\right)}$ and $\eta_{f}\left(\underline{x}_{\mathrm{p}}\right)$ are statistically independent.

Within the model of Eq.(21), the expectation and the variance of any Poisson variable being equal, it is possible to compute both the mean and the standard deviation of $f\left(\underline{x}_{\mathrm{p}}\right)$, which are written as follows $(c f[7,11])$ :

$$
\left\{\begin{array}{l}
\left\langle f\left(\underline{x}_{\mathrm{p}}\right)\right\rangle=\mathcal{G}_{0} \times \nu\left(\underline{x}_{\mathrm{p}}\right)+\left\langle\eta_{f}\right\rangle \\
\sigma_{f, \mathrm{p}}^{2}\left(\underline{x}_{\mathrm{p}}\right)=\mathcal{G}_{0}^{2} \times \nu\left(\underline{x}_{\mathrm{p}}\right)+\sigma_{\eta_{f}}^{2}
\end{array}\right.
$$

where $\sigma_{f, \mathrm{p}}\left(\underline{x}_{\mathrm{p}}\right)$ denotes the standard deviation of the gray level $f$ measured at $\underline{x}_{\mathrm{p}}$. By substituting $\nu\left(\underline{x}_{\mathrm{p}}\right)$, the following relation consequently holds:

$$
\sigma_{f, \mathrm{p}}^{2}\left(\underline{x}_{\mathrm{p}}\right)=\mathcal{G}_{0}\left(\left\langle f\left(\underline{x}_{\mathrm{p}}\right)\right\rangle-\left\langle\eta_{f}\right\rangle\right)+\sigma_{\eta_{f}}^{2} .
$$

This model predicts that, at a given pixel, the noise variance linearly depends on the expected pixel intensity. The noise variance therefore exhibits spatial fluctuations because of the pixel intensity changes, and is thus a heteroscedastic process. The next paragraphs illustrate two strategies denoted $\mathcal{S}_{1}$ and $\mathcal{S}_{2}$ which take such noise into account for the consistent elaboration of displacement resolution when real images are processed by DIC.

$\mathcal{S}_{1}$ : Stabilizing heteroscedastic noise with the GAT The first strategy consists of preprocessing the images in order to stabilize the variance; in other words to make it spatially constant, and thus to change the heteroscedastic Poisson-Gaussian noise distribution into a 


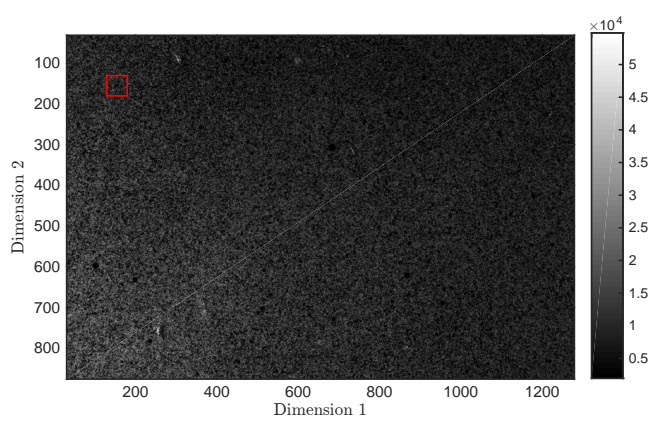

(a) Pixel mean value $\langle f\rangle$ [gray level]

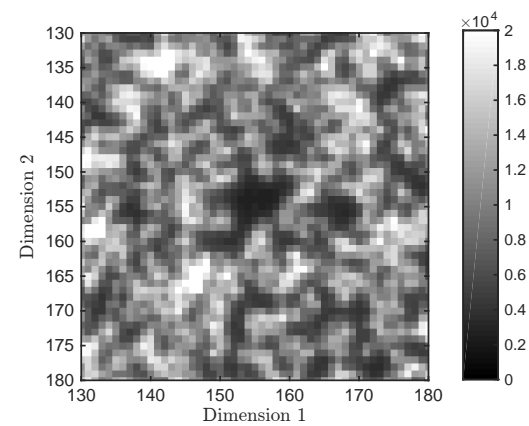

(c) Close up of pixel mean value $\langle f\rangle$ [gray level]

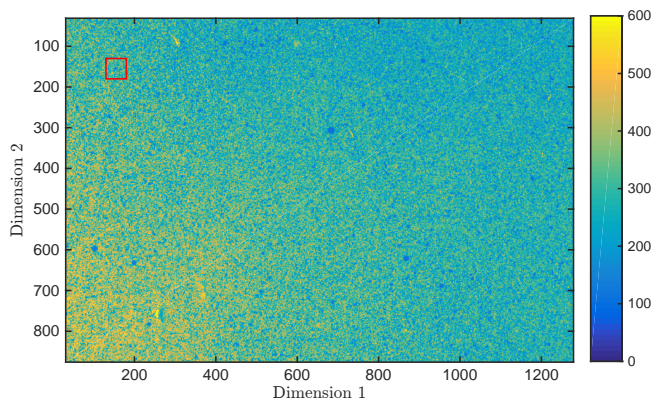

(b) Pixel standard deviation $\sigma_{f, \mathrm{p}}$ [gray level]

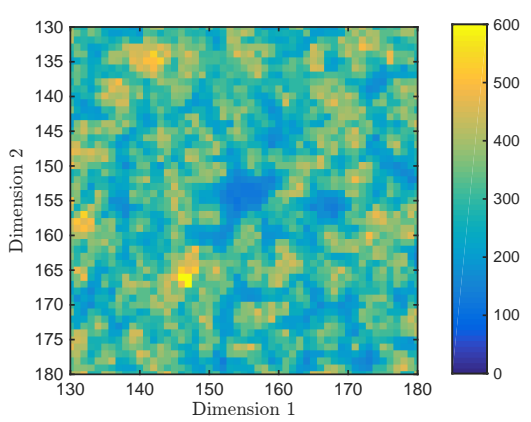

(d) Close up of pixel standard deviation $\sigma_{f, \mathrm{p}}$ [gray level]

Figure 5: Main characteristics of the statistical analysis; pixel mean value $\langle f\rangle$ (a), pixel standard deviation $\sigma_{f, \mathrm{p}}(\mathrm{b})$, their respective close-ups (c) \& (d).

homoscedastic Gaussian distribution. This is exactly the aim of the GAT (see [24] after [1]). In addition, the GAT causes the variance of the noise, and thus its standard deviation, to be uniformly equal to unity. The GAT is said to stabilize the variance and the standard deviation. With our notations, the GAT is written:

$$
f_{\mathrm{G}}\left(\underline{x}_{\mathrm{p}}\right)=\frac{2}{\mathcal{G}_{0}} \sqrt{\mathcal{G}_{0}\left(f\left(\underline{x}_{\mathrm{p}}\right)-\left\langle\eta_{f}\right\rangle\right)+\frac{3}{8} \mathcal{G}_{0}^{2}+\sigma_{\eta_{f}}^{2}}
$$

We denote here with subscript "G" any quantity (image, matrix, ...) obtained after applying the GAT.

The transformed image $f_{\mathrm{G}}\left(\underline{x}_{\mathrm{p}}\right)$ is such that its noise is approximately modeled with a Gaussian distribution of variance equal to 1 . It is also worth mentioning that the gray level is affected at any pixel. This corrected gray level is a floating point, not an integer. The same process can also be applied to image $(g)$ to define $\left(g_{\mathrm{G}}\right)$. It is worth mentioning that the GAT is a non-linear transformation which could thus introduce a bias into the displacement maps. Nevertheless, since DIC DoF each rely on several pixels of several gray levels, the order of magnitude of GAT 
effects is negligible compared to that of interpolation, for instance. By applying DIC to a pair of images $\left(f_{\mathrm{G}}, g_{\mathrm{G}}\right)$, it is therefore expected that the $\mathcal{H}_{1}$ hypothesis become satisfied, so the predictive formula can be applied with a proper value for $\sigma_{f_{\mathrm{G}}}(=1)$. At node $k$, the associated predictive resolution $\sigma_{u_{\mathrm{G}}}^{p}$ is written

$$
\begin{aligned}
\sigma_{u_{\mathrm{G} \ell}\left(\underline{x}_{\mathrm{k}}\right)}^{p} & =\sqrt{\left[\boldsymbol{M}_{\mathrm{G}}^{-1}+\boldsymbol{M}_{\mathrm{G}}^{-1} \boldsymbol{L}_{\mathrm{G}} \boldsymbol{P} \boldsymbol{P}^{T} \boldsymbol{L}_{\mathrm{G}}^{T} \boldsymbol{M}_{\mathrm{G}}^{-1}\right]_{l l}} \times \sigma_{f_{\mathrm{G}}} \\
& =\sqrt{\left[\boldsymbol{M}_{\mathrm{G}}^{-1}+\boldsymbol{M}_{\mathrm{G}}^{-1} \boldsymbol{L}_{\mathrm{G}} \boldsymbol{P} \boldsymbol{P}^{T} \boldsymbol{L}_{\mathrm{G}}^{T} \boldsymbol{M}_{\mathrm{G}}^{-1}\right]_{l l}}
\end{aligned}
$$

where $\ell$ corresponds to the displacement component on which the resolution is predicted, and $l$ to the appropriate degree of freedom. The DIC-tangent operator $\boldsymbol{M}_{\mathrm{G}}$ is defined following Eq.(5):

$$
\left[\boldsymbol{M}_{\mathrm{G}}\right]=\boldsymbol{L}_{\mathrm{G}} \boldsymbol{L}_{\mathrm{G}}^{T}, \text { with } \forall(i, j) \in\left\{1, N_{\mathrm{DoF}}\right\} \times\left\{1, N_{p}\right\},\left[\boldsymbol{L}_{\mathrm{G}}\right]_{i, j}=\underline{\nabla} f_{\mathrm{G}}\left(\underline{x}_{\mathrm{j}}\right) \underline{\varphi}_{i}\left(\underline{x}_{\mathrm{j}}\right) .
$$

Hence, the GAT is applied to the $2 \times N_{\text {img }}$ images $\left(f_{t}\right)_{1 \leq t \leq N_{\text {img }}}$ and $\left(g_{t}\right)_{1 \leq t \leq N_{\text {img }}}$ to obtain their corrected version, denoted $\left(f_{\mathrm{G}, t}\right)_{1 \leq t \leq N_{\mathrm{img}}}$ and $\left(g_{\mathrm{G}, t}\right)_{1 \leq t \leq N_{\mathrm{img}}}$. The image gradient $\left(\underline{\nabla} f_{\mathrm{G}}\right)$ refers here to the gradient of an updated average image $\left\langle f_{\mathrm{G}}\right\rangle$, which can be considered as noiseless. Hypothesis $\mathcal{H}_{3}$ is thus satisfied. Finally, we can elaborate an empirical calculation of the standard deviation of the displacement $\sigma_{u_{\mathrm{G}}}^{e}$ after applying the DIC to the corrected pairs of images

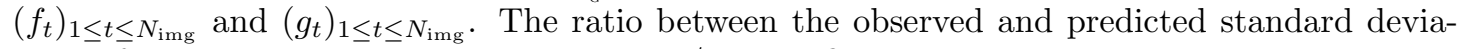
tions of the vertical displacement $\rho_{\mathrm{G}}=\sigma_{u_{\mathrm{G}, 2}}^{e} / \sigma_{u_{\mathrm{G}, 2}}^{p}$ is finally computed in order to highlight the differences between raw and updated image processing using the GAT. It is worth mentioning that the GAT has recently been introduced in photomechanics, as in [14] in the case of the Grid Method, but has never been employed in DIC before, to the best of the authors' knowledge.

$\mathcal{S}_{2}$ : Relaxing hypothesis $\mathcal{H}_{1}$ The second strategy consists of considering the hypothesis $\overline{\mathcal{H}}_{1}$, the relaxed form of $\mathcal{H}_{1}$, which takes into account 0 -mean Gaussian image noise of standard deviation $\sigma_{f, \mathrm{p}}\left(\underline{x}_{\mathrm{p}}\right)$ which are spatially dependent. This is here directly defined thanks to the pixel gray level standard deviation, following Eq.(17). The covariance matrices $\boldsymbol{\Sigma}_{\boldsymbol{n}_{f}}$ and $\boldsymbol{\Sigma}_{\boldsymbol{n}_{g}}$ of the image noise are diagonal and composed of the terms :

$$
\forall i \in\left\{1, \ldots, N_{p}\right\},\left[\boldsymbol{\Sigma}_{\boldsymbol{n}_{f}}\right]_{i i}=\sigma_{f, \mathrm{p}}\left(\underline{x}_{\mathrm{i}}\right) \text { and }\left[\boldsymbol{\Sigma}_{\boldsymbol{n}_{g}}\right]_{i i}=\sigma_{g, \mathrm{p}}\left(\underline{x}_{\mathrm{i}}\right) .
$$

According to Eq.(15), the predictive resolution $\bar{\sigma}_{u}^{p}$ and the associated ratio $\bar{\rho}=\frac{\sigma_{u_{2}}^{e}}{\bar{\sigma}_{u_{2}}^{p}}$ can also be computed.

\subsubsection{Results and discussion}

Using the $N_{\text {img }}$ images which have been acquired ( $c f$ Sec.(3.1)), the experimental determination of the parameters introduced in Eq.(23) is possible. As illustrated in Fig.(6a), we obtain with robust linear regression

$$
\left\{\begin{array}{l}
\mathcal{G}_{0}=10.02 \\
\sigma_{\eta_{f}}^{2}-\mathcal{G}_{0} \times\left\langle\eta_{f}\right\rangle=-1.12 \times 10^{4}
\end{array} .\right.
$$

Fig.(6) illustrates the two maps of sample variance against the sample mean value of $\left(f_{t}\right)_{1 \leq t \leq N_{\mathrm{img}}}$

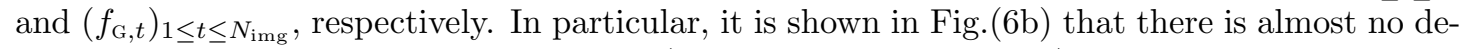
pendency any longer between the variance (or the standard deviation) of the gray level intensity and the mean value of this gray level intensity at each pixel position. Images $\left(f_{\mathrm{G}}\right)$ can thus be considered as being affected by homoscedastic noise, and the same conclusion can be drawn for $\left(g_{\mathrm{G}}\right)$. 


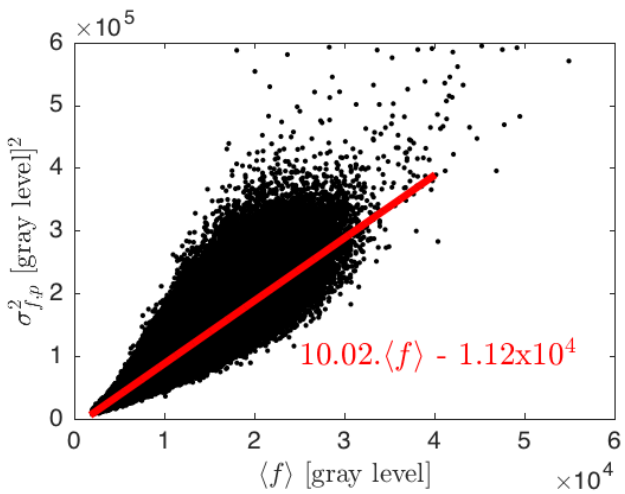

(a)

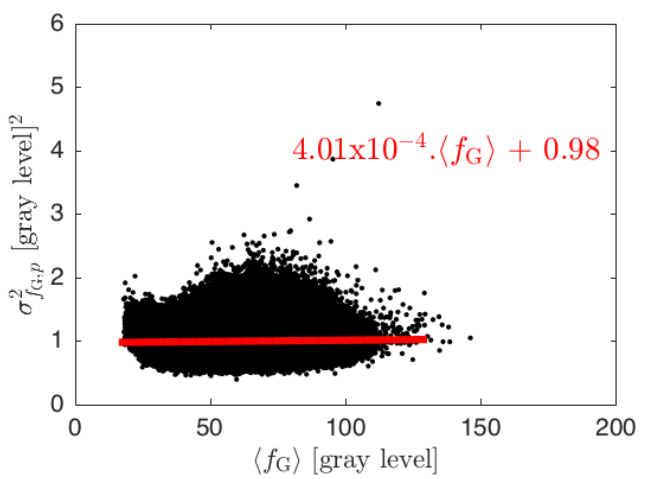

(b)

Figure 6: Plots of sample variance against the sample mean value, in gray level, at each pixel position: (a) before GAT $\left(\langle f\rangle, \sigma_{f, \mathrm{p}}^{2}\right)$ and (b) after GAT $\left(\left\langle f_{\mathrm{G}}\right\rangle, \sigma_{f_{\mathrm{G}}, \mathrm{p}}^{2}\right)$. On both maps, robust linear fits are shown in red, with their related equations.

Ratios $\bar{\rho}=\frac{\sigma_{u_{2}^{e}}}{\bar{\sigma}_{2}^{p}}$ and $\rho_{\mathrm{G}}=\frac{\sigma_{u_{\mathrm{G}, 2}}^{e}}{\sigma_{u_{\mathrm{G}, 2}}^{p}}$ between observations and predictions of vertical displacement resolution are illustrated in Fig.(7). These two ratios are similar, in terms of spatial distribution (see maps in Fig.(7a\&c)) as well as in terms of dispersion (see histograms in Fig.(7b\&d)). This means that the two strategies proposed here lead to similar results. Relaxing hypothesis $\mathcal{H}_{1}$ seems to be a method with a reduced computational cost, since it only requires the calculation of the covariance matrix $\boldsymbol{\Sigma}_{\boldsymbol{n}}$, whereas GAT processing has to be applied to all raw images. Nevertheless, working with images corrupted by white Gaussian noise of 0 -mean and a standard deviation set to 1 standardizes the input images of the DIC process. Consequently, the noise at each pixel is identically weighted, independently of the pixel gray level, and therefore plays an identical role within the whole DIC procedure.

Note that the color-bars used in Fig. (7a\&c) are the same as those already employed in Fig.(3c). This emphases the slight reduction in ratio dispersion, extremal values now being closer to 1 . Fig.(7b\&d) also highlight the fact that the histogram of the ratios between predicted and observed quantities have been sharpened by taking into account the heteroscedasticity of the sensor noise.

As an intermediate conclusion, considering the heteroscedasticity of the sensor noise did not radically enhance the applicability of the predictive formula, since the mean values of the ratios between empirical and predictive displacement resolutions before and after the two proposed methods remain similar. Nevertheless, the predictions of this resolution present better properties after execution. On the one hand, the ratio between empirical and predicted values of the displacement resolution is less dependent on illumination characteristics. Indeed, the spatial fluctuation of the illumination is no longer observable, see Fig.(7a\&c)). On the other hand, the histograms Fig. (7b\&d) are sharper; more specifically, the right tails are smaller. However, the mean value is still over $60 \%$ higher than the expected one, thus indicating the presence of another phenomenon. 


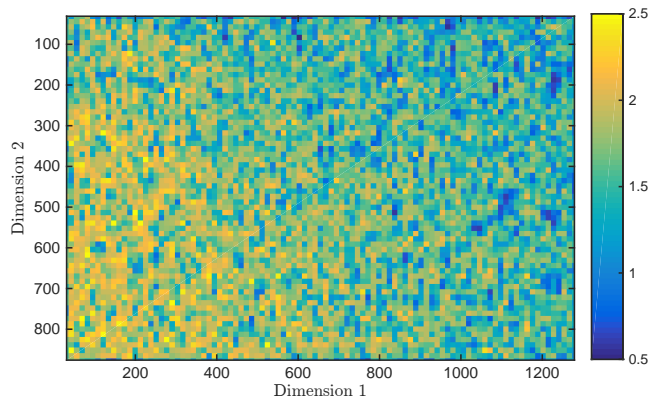

(a) Map of ratio $\bar{\rho}[-]$

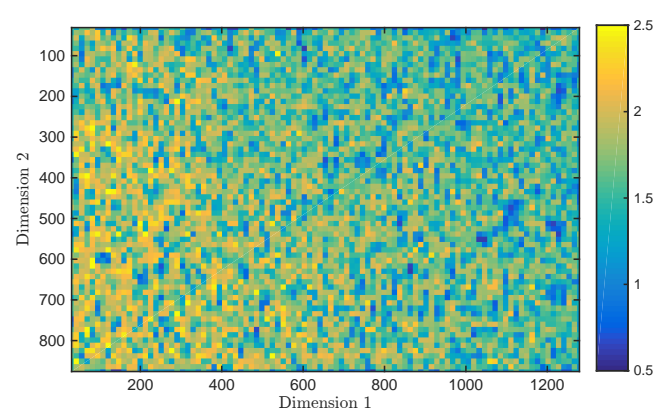

(c) Map of ratio $\rho_{\mathrm{G}}[-]$

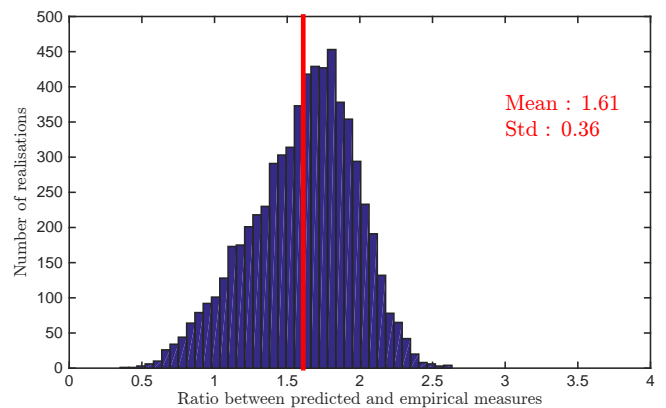

(b) Histogram of ratio $\bar{\rho}[-]$

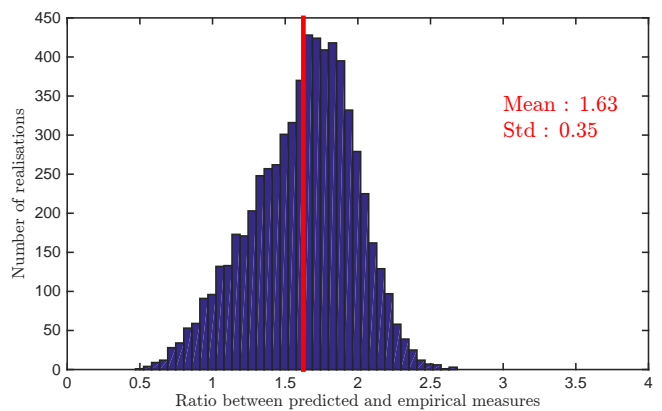

(d) Histogram of ratio $\rho_{\mathrm{G}}[-]$

Figure 7: Figures depicting the ratios between empirical and predicted resolutions of vertical displacement. The map (a) and the histogram (b) illustrate the ratio $\bar{\rho}$, which is computed between the empirical resolution and the predicted one, once the hypothesis $\mathcal{H}_{1}$ has been relaxed into $\overline{\mathcal{H}}_{1}$. The map (c) and the histogram (d) present the ratio $\rho_{\mathrm{G}}$ between observation and prediction of the resolution, in the case of the GAT having been applied to pre-process the images.

\subsection{Taking RBM into account}

\subsubsection{Experimental evidence of micro-movements}

The present study also highlights another important root of the apparent displacement resolution. For this purpose, the vertical displacements at four points defined in Fig.(1b) and obtained for each DIC realization $\left(f_{t}, g_{t}\right)_{1 \leq t \leq N_{\text {img }}}$ are shown in Fig.(8). A spatial correlation between the displacements of the four points is here clearly visible. This is due to the fact that Rigid Body Motion (RBM) occurred during acquisition, and was thus recovered by the DIC process. Such micro-movements impair the estimation at a given pixel of both the expected value (Eq.(16)) and the sensor noise standard deviation (Eq.(17)). Indeed, in the case of micro-movements, the measured values at a given pixel over time are no longer instances of the same random process. A consequence of this micro-movement on the predictive formula is thus the non-consistency of hypothesis $\mathcal{H}_{1}$ with the experimental data. It should be pointed out that such micro-movements 


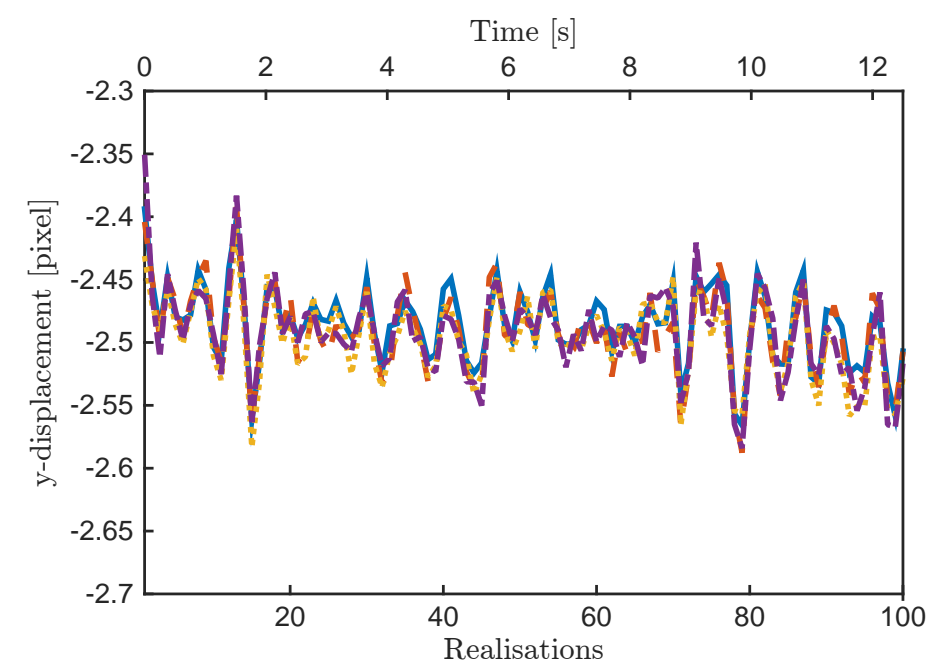

Figure 8: Variation in vertical displacement at points $1-4$ (cf Fig.(1b)) for $N_{\text {img }}$ images.

are always present in our experiments, whatever the machine or the room used, even though they are carried out in a laboratory environment. They are due to vibrations transmitted by the concrete slab on which both the tensile machine and the camera tripod rest.

In the wake of recent progress originally introduced for the Grid Method [41, 14, 42, 43], the last part of this section proposes a strategy focused on the strong reduction in displacement fluctuation by adding pre-processing to the image series before applying the DIC program. This pre-processing allows us to eliminate the effect of RBM within the raw images, and thus enables the sensor noise properties to be correctly estimated, and eventually $\mathcal{H}_{1}$ to be satisfied.

Since this pre-processing can be applied only for grid images in the current version proposed in [41], special attention is also paid here to applying DIC to the grid images. This is detailed in Appendix (B). The two pre-processing strategies discussed above (namely (i) changing heteroscedastic noise into homoscedastic noise $\left(\mathcal{S}_{1}\right)$ or relaxing Hypothesis $\mathcal{H}_{1}$ into $\overline{\mathcal{H}}_{1}\left(\mathcal{S}_{2}\right)$, and (ii) removing the effect of micro-movements on the images) are independent. Consequently, different sets of images are built up and studied when DIC is applied to them.

\subsubsection{Non-Signal Reduction Method - basics}

A method is proposed in [41] to eliminate the effect of residual vibrations on the time-variations in intensity at a given pixel of a periodic pattern like a grid. Grids are characterized by their pitch, denoted $p$. A brief reminder of this technique is given here. Noting $h(x, y, t)$ the gray-level intensity of the pixel $\underline{x}_{\mathrm{p}}=(x, y)$ in the $t$-th image, we define a non-local average as follows:

$$
H(x, y, t)=\frac{1}{N_{\mathrm{NRSR}}} \sum_{k, l} w_{k, l} . h(x+k p, y+l p, t)
$$

where $(k, l)$ spans a set around $(0,0)$ of cardinality $N_{\mathrm{NRSR}}$ and $w_{k, l}$ are weights summing to 1 . The weights are chosen with an exponential decay to the reference pixel at $(x, y)$, as proposed in [41]. Averaging removes the intensity noise; hence the time-variations of $H$ correspond to the 
time-variations of $h$ solely caused by the vibrations (as proved in [41]). Consequently $h(x, y, t)-$ $H(x, y, t)$ is the noise component. Noting $\langle h(x, y)\rangle_{t}$ the time-average of $h(x, y, t),\langle h(x, y)\rangle_{t}+$ $h(x, y, t)-H(x, y, t)$ is the gray-level intensity at pixel $(x, y)$ in the $t$-th image, corrected from the fluctuations caused by vibrations, but still affected by the signal-dependent, heteroscedastic noise. This quantity is the output of the NRSR algorithm.

\subsubsection{Reducing the rigid body motion effect directly in raw images - NRSR}

It is worth noting that the NRSR method uses periodic patterns like grids. Consequently, DIC is applied here to imaged grids, which present similar properties to the imaged speckles used previously, see Appendix (B). RBM occurring during grid image acquisition is shown in Fig.(9a). The DIC recovers the vertical displacement $\mathrm{u}_{2}$ at four points (same location as in Fig.(1b)). This displacement is plotted for each of the $N_{\text {img }}$ DIC realizations on the image pairs

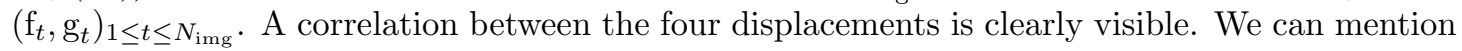
that such measurements are associated with the test conditions, but they are repeatable in our laboratory. For instance Fig.(8) illustrates the same quantities (displacement at four points) but for a different test; the grid has been imaged in this case, and the order of magnitude is consistent $( \pm 0.1$ [pixel] $= \pm 4[\mu \mathrm{m}])$. Moreover, such RBM occurs at low frequencies: motion blur in the images thus does not occur. In the case of a G-DIC study, kinematics could be defined by directly including RBM. The output displacement maps would thus directly avoid the RBM effects, as would their resolution. The objective here is to master the sensor noise and also to validate the predictive formula, and RBM should as a consequence be removed directly within the raw images.

The NRSR algorithm is here applied to the $N_{\text {img }}$ pairs of images $\left(\mathrm{f}_{t}, \mathrm{~g}_{t}\right)_{1 \leq t \leq N_{\mathrm{img}}}$ to elaborate a set of RBM-free pairs of images $\left(\mathrm{f}_{\mathrm{N}, t}, \mathrm{~g}_{\mathrm{N}, t}\right)_{1 \leq t \leq N_{\mathrm{img}}}$. DIC is then performed on these pairs of images, defining $\underline{u}_{N}$. Fig.(9b) represents $u_{N, 2}$ at the same four points for each DIC realization. No correlation between the four displacements is visible any more, which means that the RBM that occurred during the experiment has been correctly removed from the raw images. The inset in Fig.(9b) presents the same results as in Fig.(9b) but at a reduced scale. Uncorrelated fluctuations between the four displacements are now observable. These fluctuations are the consequence of the sensor noise.

Thanks to the NRSR pre-processing, images $\left(\mathrm{f}_{\mathrm{N}, t}\right)_{1 \leq t \leq N_{\mathrm{img}}}$ as well as images $\left(\mathrm{g}_{\mathrm{N}, t}\right)_{1 \leq t \leq N_{\mathrm{img}}}$ are now free of RBM and their noise can be reduced to sensor noise. The effect of this pre-processing on displacement resolution will now be presented.

First, the standard deviation of the noise $\sigma_{\mathrm{f}}$ can be computed from the updated reference images following Eq.(16-18), and predictive formulas are also employed to predict the measurement resolution. The predicted standard deviation of the vertical displacement $\sigma_{\mathrm{u}_{\mathrm{N}, 2}}^{p}$ can thus be elaborated. Second, the stack of $N_{\mathrm{img}}$ DIC outputs enables the calculation of an empirical standard deviation of the vertical displacement $\sigma_{\mathrm{u}_{\mathrm{N}, 2}}^{e}$.

Fig.(10) presents the ratio between empirical and predicted displacement resolutions, when DIC is applied to pre-processed NRSR images. This ratio is mapped in Fig.(10a) and its histogram is illustrated in Fig.(10b). The bright spots in map Fig.(10a) correspond to the locations of grid defects. Indeed, because of the lack of regularity in the grid pattern, the NRSR algorithm fails to completely remove rigid body motion. Consequently, at these locations the images are still corrupted by micro-movements, which lead to a high empirical standard deviation. Nevertheless, except for these outliers, the ratio distribution is smoother here than in the previous case discussed in Section 3.3. First, the ratio is closer to 1; second, the histogram of this ratio has been sharpened thanks to the NRSR pre-processing. 


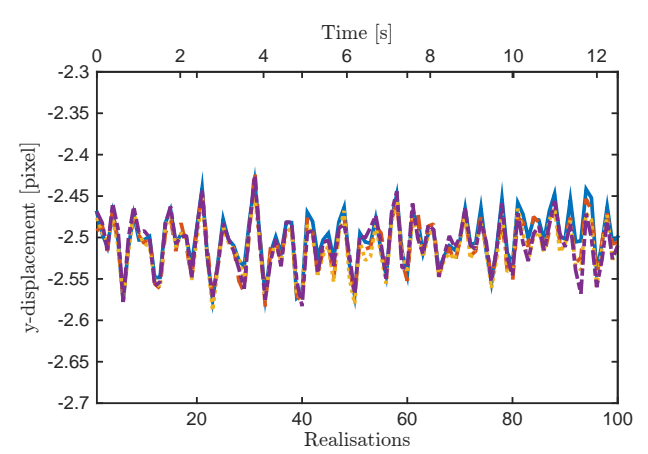

(a) Variation in vertical displacement at points $1-4$ (location defined in Fig.(1a)) for $N_{\text {img images - Before }}$ NRSR

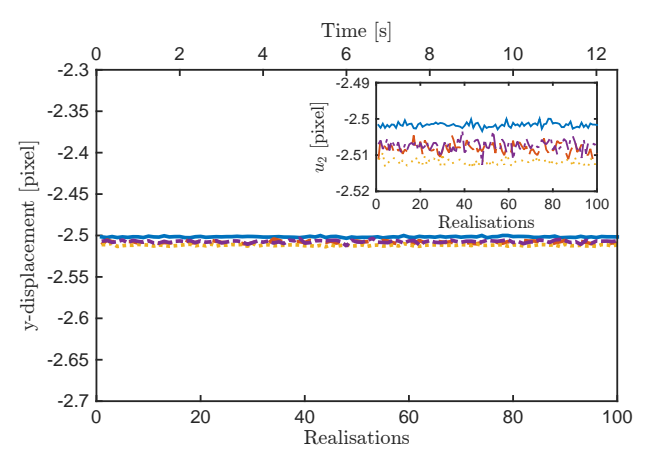

(b) Variation in vertical displacement at points $1-4$ (location defined in Fig.(1b)) for $N_{\text {img images }- \text { After }}$ NRSR

Figure 9: Figures highlighting the rigid body motion occurring during the acquisition of $2 \times N_{\text {img }}$

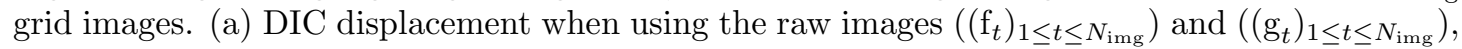
(b) same quantities, but the DIC is here applied after NRSR pre-processing, i.e., to images $\left(\left(\mathrm{f}_{\mathrm{N}, t}\right)_{1 \leq t \leq N_{\mathrm{img}}}\right)$ and $\left(\left(\mathrm{g}_{\mathrm{N}, t}\right)_{1 \leq t \leq N_{\mathrm{img}}}\right)$. The inset in (b) shows the same quantities as (b) with a reduced scale.

\subsubsection{Accounting for both the RBM and the correct sensor noise model}

Images $\left(\mathrm{f}_{\mathrm{N}, t}\right)_{1 \leq t \leq N_{\mathrm{img}}}$ as well as images $\left(\mathrm{g}_{\mathrm{N}, t}\right)_{1 \leq t \leq N_{\mathrm{img}}}$ have been cleaned of the RBM effect but they still inherit the heteroscedastic property of their noise from the camera sensor. As explained above, there are two strategies, $\mathcal{S}_{1}$ and $\mathcal{S}_{2}$, to take into account the heteroscedastic nature of the noise. $\mathcal{S}_{1}$ consists of applying GAT pre-processing to these images in order to build up images $\left(\mathrm{f}_{\mathrm{N}-\mathrm{G}, t}\right)_{1 \leq t \leq N_{\mathrm{img}}}$ and $\left(\mathrm{g}_{\mathrm{N}-\mathrm{G}, t}\right)_{1 \leq t \leq N_{\mathrm{img}}}$, in which the noise then presents homoscedastic properties, $c f$ Fig.(11a-b). DIC is then applied to these images, enabling the displacement $\underline{\mathrm{u}}_{\mathrm{N}-\mathrm{G}}$ to be recovered. The stack of images acquired at both steps of the experiment allows us to build up an empirical displacement resolution $\sigma_{\mathrm{u}_{\mathrm{N}-\mathrm{G}, 2}}^{e}$ once DIC has been applied. It also enables the calculation of image noise properties and thus, thanks to the predictive formulas, a prediction of this measurement, $\sigma_{\mathrm{u}_{\mathrm{N}-\mathrm{G}, 2}}^{p}$, can also be elaborated. Finally, the ratio between these two resolutions is calculated $\rho_{\mathrm{N}-\mathrm{G}}=\sigma_{\mathbf{u}_{\mathrm{N}-\mathrm{G}, 2}}^{e} / \sigma_{\mathbf{u}_{\mathrm{N}-\mathrm{G}, 2}}^{p}$ to observe any deviation from one.

Coefficients $\mathcal{G}_{0}$ and $\sigma_{\eta_{f_{\mathrm{N}}}}^{2}-\mathcal{G}_{0}\left\langle\eta_{f_{\mathrm{N}}}\right\rangle$ involved in the GAT algorithm are here identified using a robust linear regression, from the data illustrated in Fig.(11a). This noise covariance is then used to compute the predictive resolution $\bar{\sigma}_{\mathrm{u}_{\mathrm{N}, 2}}^{p}$ as well as the ratio $\bar{\rho}_{\mathrm{N}}$ between the observed vertical displacement resolution $\sigma_{\mathrm{u}_{\mathrm{N}, 2}}^{e}$ and the prediction.

Remark 3.1 The identification of the camera sensor noise parameters $\mathcal{G}_{0}$ and $\sigma_{\eta_{f}}^{2}-\mathcal{G}_{0}$ is here performed using a series of images describing the same stage. It is worth noting that these parameter values correspond to the camera sensor features, and thus to those identified with the same camera as in [14, 41]. This simple analysis can of course be carried out for any kind of camera if the parameters are not given by the manufacturer.

Strategy $\mathcal{S}_{2}$ consists of relaxing hypothesis $\mathcal{H}_{1}$ into $\overline{\mathcal{H}}_{1}$, by defining the covariance $\boldsymbol{\Sigma}_{\boldsymbol{n}_{\mathrm{N}}}$ of the RBM-free image noise which takes into account the particular properties of the sensor noise. Following Eq.(28) with the images cleaned of the RBM effect, we have 


$$
\forall i \in\left\{1, . ., N_{p}\right\},\left[\boldsymbol{\Sigma}_{\boldsymbol{n}_{\mathrm{N}}}\right]_{i i}=\sigma_{f_{\mathrm{N}}, \mathrm{p}}\left(\underline{x}_{\mathrm{i}}\right), \text { with } \sigma_{f_{\mathrm{N}}, \mathrm{p}}\left(\underline{x}_{\mathrm{i}}\right)=\sqrt{\frac{1}{N_{\mathrm{img}}-1} \sum_{t=1}^{N_{\mathrm{img}}}\left\{\left(f_{\mathrm{N}, t}-\left\langle f_{\mathrm{N}}\right\rangle\right)^{2}\right\}} .
$$

Fig.(12) illustrates the ratios $\rho_{\mathrm{N}-\mathrm{G}}$ and $\bar{\rho}_{\mathrm{N}}$ between the empirical and predicted resolutions, now taking into account both RBM and a correct model for the sensor noise. Both ratios present exactly the same properties, in terms of spatial distribution and dispersion. Moreover, there is now quite a homogeneous spatial distribution of these ratios, and they are very close to 1 . The histograms shown in Figs.(12b\&d) have also been strongly sharpened thanks to the correct sensor noise model that is now assumed in both predictive resolutions. This result means that the predictive formulas given in Eq.(13) and Eq.(15) are experimentally verified if the noise which affects the images is correctly modeled. 


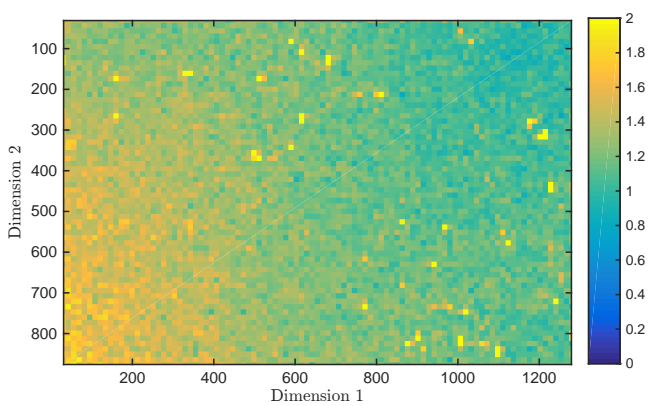

(a) Map of ratio $\rho_{\mathrm{u}_{\mathrm{N}}}[-]$

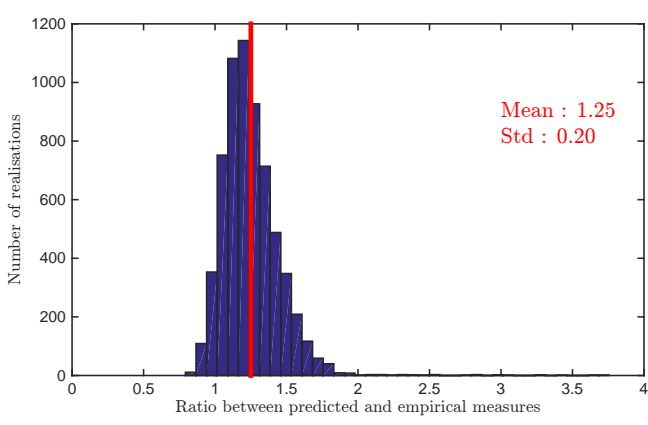

(b) Histogram of ratio $\rho_{\mathrm{u}_{\mathrm{N}}}[-]$

Figure 10: Ratio between empirical and predicted standard deviation of the vertical displacement when DIC is applied after NRSR alone, $\rho_{\mathrm{u}_{\mathrm{N}}}=\sigma_{\mathrm{u}_{\mathrm{N}, 2}}^{e} / \sigma_{\mathrm{u}_{\mathrm{N}, 2}}^{p}$.

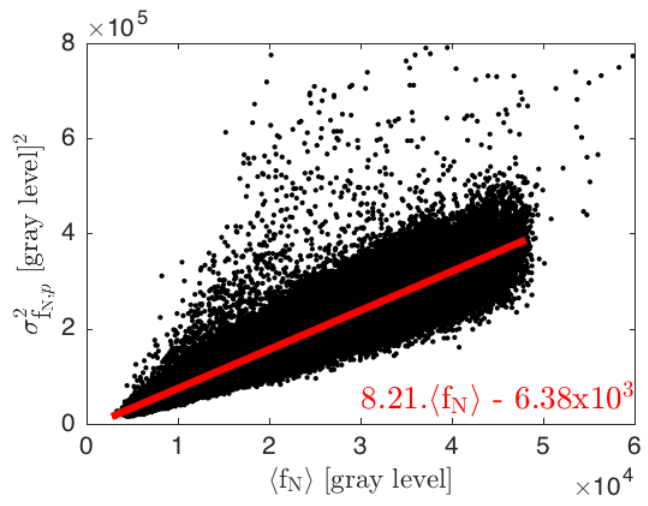

(a)

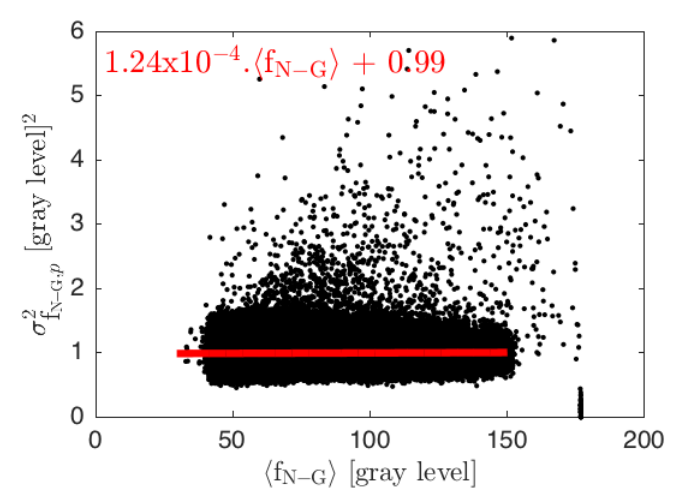

(b)

Figure 11: Maps of variance against mean value, in gray level, at each pixel position: (a) after $\operatorname{NRSR}\left(\left\langle f_{\mathrm{N}}\right\rangle, \sigma_{f_{\mathrm{N}} \mathrm{p}}^{2}\right)$ and (b) after NRSR and GAT $\left(\left\langle f_{\mathrm{N}-\mathrm{G}}\right\rangle, \sigma_{\mathrm{N}_{\mathrm{N} \mathrm{G}}, \mathrm{p}}^{2}\right)$. On the two maps, linear fits are shown in red, with their related equation. 


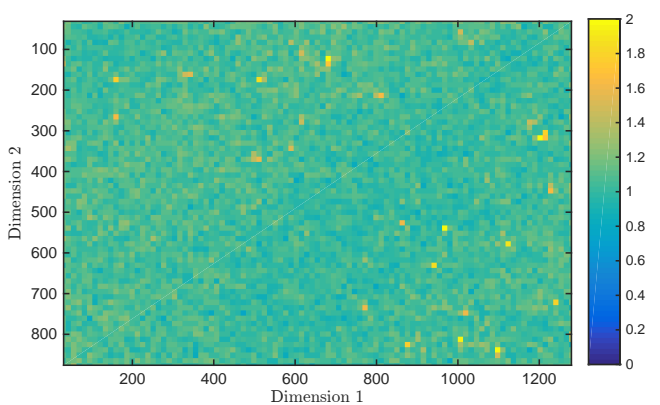

(a) Map of ratio $\bar{\rho}_{\mathrm{N}}[-]$

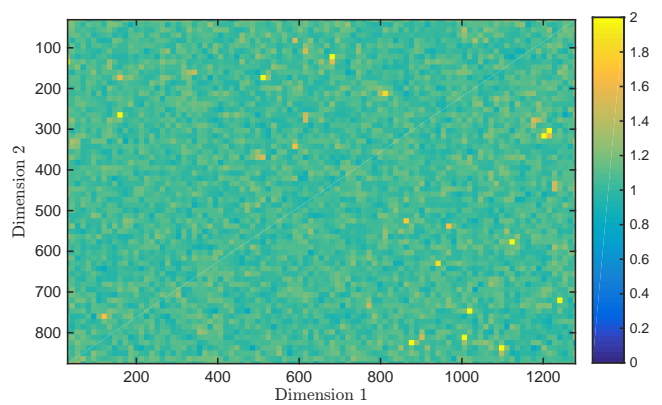

(c) Map of ratio $\rho_{\mathrm{N}-\mathrm{G}}[-]$

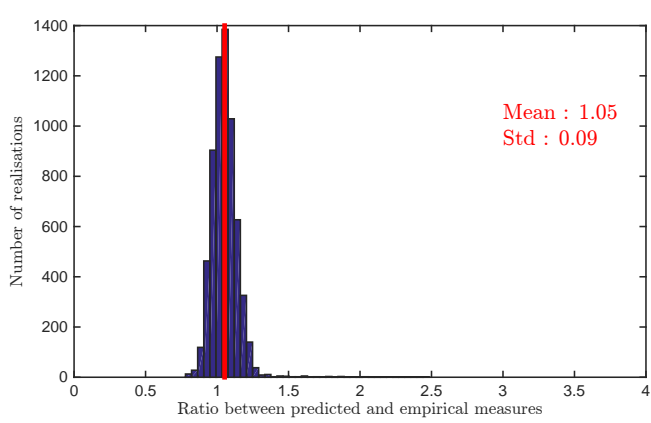

(b) Histogram of ratio $\bar{\rho}_{\mathrm{N}}[-]$

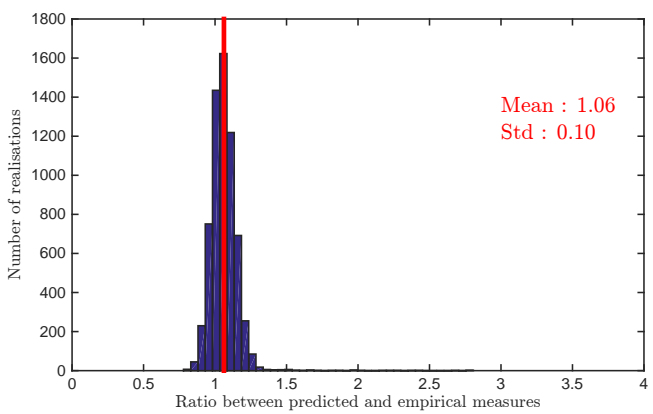

(d) Histogram of ratio $\rho_{\mathrm{N}-\mathrm{G}}[-]$

Figure 12: Ratios between predicted and observed standard deviation of the vertical displacement when DIC is applied after NRSR and enabling the correct sensor noise model. The map in (a) and the histogram in (b) illustrate the ratio $\bar{\rho}_{\mathrm{N}}$, which is computed between the empirical and the predicted resolution, after NRSR pre-processing of the images and once the hypothesis $\mathcal{H}_{1}$ has been relaxed into $\overline{\mathcal{H}}_{1}$. The map in (c) and the histogram in (d) present the ratio $\bar{\rho}_{\mathrm{N}-\mathrm{G}}$ between observation and prediction of the resolution, in the case of both the NRSR and the GAT having been applied to pre-process the images. 


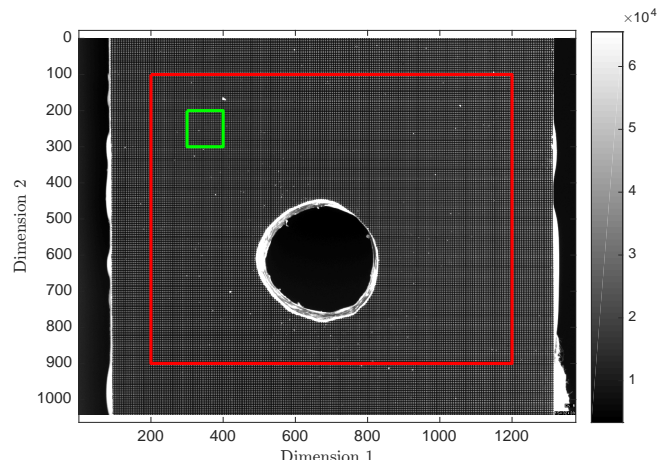

(a) Open-hole specimen [gray level]

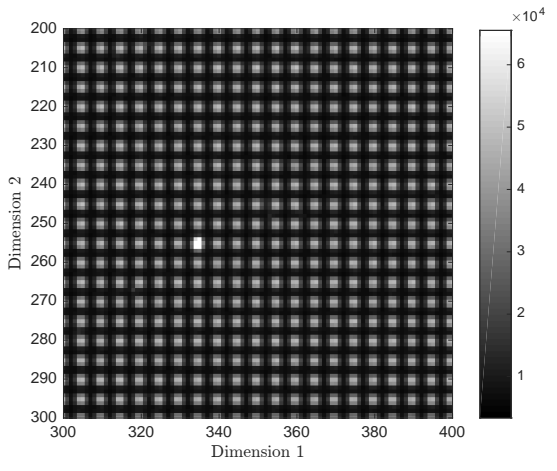

(b) Close-up (green box in (a)) [gray level]

Figure 13: Open hole specimen. (a) Zone observable with the camera: the red box in the Region of Interest. (b) Close-up: green box in (a).

\section{Experimental validation: tensile test on a open-hole spec- imen}

In this section, the formula is validated via a more realistic case: an open-hole specimen subjected to a tensile test. Once loaded, a heterogeneous strain distribution occurs around the hole, enabling us to validate the resolution predictive formula in a more complicated case. First, the set-up and the experimental methods are detailed. Special attention is paid to describing the two DIC approaches that are implemented here: Local and Global DIC are used; and the predictive formula is then validated in both cases.

\subsection{Experimental set-up and methods}

The same universal tensile machine as in section 3.1 is used here. The specimen is now a plate with an open hole. It is illustrated in Fig.(13). A grid is deposited on the specimen following [33]. This grid is imaged using the same camera as in Section 3.1. The RoI, shown as a red rectangle in Fig.(13), is here a region which is $40 \times 32.15[\mathrm{~mm}]^{2}$ in size, discretized into $1000 \times 800$ [pixel $]^{2}$. A close up of Fig.(13b) illustrates the imaged grid.

During the test, the speckle is illuminated using the same lightning system as in Section 3.1, but several light sources are utilized here in order to ensure an almost spatially uniform illumination.

The specimen is clamped in the universal tensile machine. Before applying any loading, 100

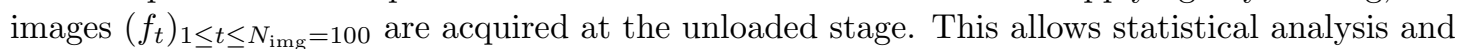
the correct acquisition of the noise corrupting the images. These images $\left(f_{t}\right)_{1 \leq t \leq N_{\mathrm{img}}}$ define the reference state. A loading force of $2500[\mathrm{~N}]$ is then applied to the specimen and another set of 100 images $\left(g_{t}\right)_{1 \leq t \leq N_{\text {img }}}$ is acquired. They define the current state.

The displacement maps of the surface specimen are illustrated in Fig.(14). Because of the hole, the horizontal (resp. vertical) displacement ranges from 0 to 1 [pixel] (resp. from -9.2 to -8.6 [pixel]). The sub-pixel part of both components of the displacement is thus non-constant over the specimen.

First, the images are pre-processed using the NRSR algorithm, see Section 3.4, to eliminate 


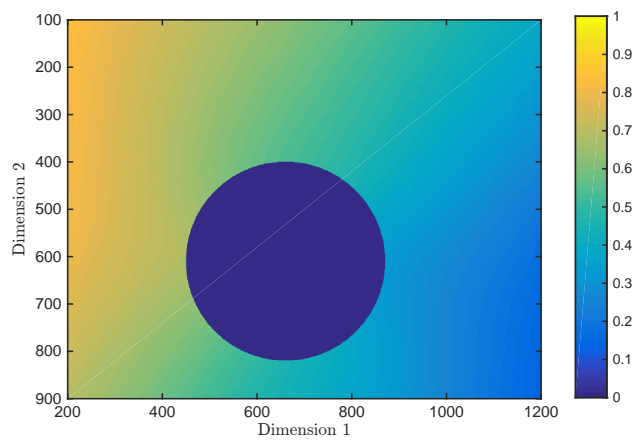

(a) Vertical displacement [pixel]

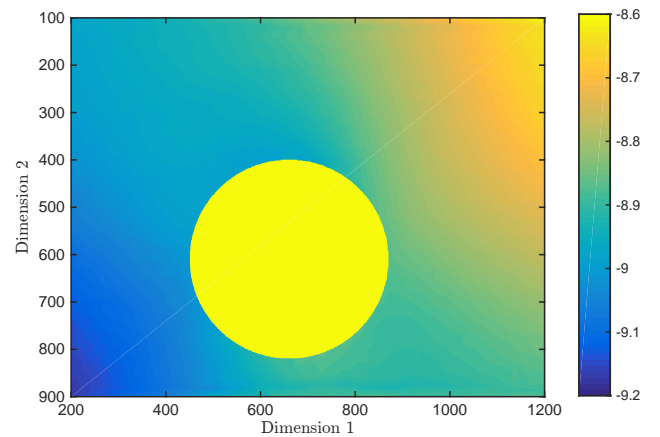

(b) Horizontal displacement [pixel]

Figure 14: Displacements maps with tensile loading set to $2500[\mathrm{~N}]$.

RBM within each set of images $\left(f_{t}\right)_{1 \leq t \leq N_{\mathrm{img}}}$ and $\left(g_{t}\right)_{1 \leq t \leq N_{\mathrm{img}}}$. Strategy $\mathcal{S}_{2}$, i.e., relaxing hypothesis $\mathcal{H}_{1}$ to $\overline{\mathcal{H}}_{1}$ and taking into account image noise that evolves spatially, is chosen here. As illustrated in Section 3.3 strategies $\mathcal{S}_{1}$ and $\mathcal{S}_{2}$ offer similar results, a property which is also verified here. Nevertheless, we deliberately develop only strategy $\mathcal{S}_{2}$, because it involves the more complex predictive formula from Eq.(15) and reveals the ease of applicability for both Land G-DIC cases; and finally for the sake of paper's readability. Indeed, for each pair of images $\left(f_{t}, g_{t}\right)_{1 \leq t \leq N_{\text {img }}}$, both Local and Global DIC are performed in order to recover the displacement maps. L- and G-DIC are implemented as follows:

L-DIC implementation The displacement is calculated for a 10 [pixel] pitch grid, and the subsets are windows of size $\ell^{\omega}=20$ [pixel]. The usual SSD criterion is used here to circumvent the updating of the proposed predictive formula. Using the ZNSSD criterion, for instance, would lead to updating the DIC tangent operator, i.e., the $\boldsymbol{M}$ matrix definition given in Eq.(5). In this work, the L-DIC kinematics are based on the first-order displacement functions. For each subset $\omega$ of center $\underline{x}_{0}^{\omega}$, displacement $\underline{u}^{\mathrm{L}}$ is thus defined through the displacement of the center of the subset (2 DoF), and the first order of displacement gradients:

$$
\forall \underline{x}_{\mathrm{p}} \in \omega, \underline{u}^{\mathrm{L}}\left(\underline{x}_{\mathrm{p}}, \lambda^{\omega}\right)=\sum_{i=1}^{6} \lambda_{i} \underline{\varphi}_{i}^{\mathrm{L}}\left(\frac{1}{2}+\frac{\underline{x}_{\mathrm{p}}-\underline{x}_{0}^{\omega}}{\ell}\right),
$$

with

$$
\begin{aligned}
& \forall \underline{x}, \underline{\varphi}_{1}^{\mathrm{L}}(\underline{x})=\underline{e}_{1}, \underline{\varphi}_{2}^{\mathrm{L}}(\underline{x})=\underline{e}_{2}, \underline{\varphi}_{3}^{\mathrm{L}}(\underline{x})=\left(\underline{x} \cdot \underline{e}_{1}\right) \underline{e}_{1}, \underline{\varphi}_{4}^{\mathrm{L}}(\underline{x})=\left(\underline{x} \cdot \underline{e}_{2}\right) \underline{e}_{2}, \underline{\varphi}_{5}^{\mathrm{L}}(\underline{x})=\left(\underline{x} \cdot \underline{e}_{1}\right) \underline{e}_{2} \\
& \text { and } \underline{\varphi}_{6}^{\mathrm{L}}(\underline{x})=\left(\underline{x} \cdot \underline{e}_{2}\right) \underline{e}_{1} \text {. }
\end{aligned}
$$

Using the $N_{\mathrm{img}}$ L-DIC realizations, an empirical displacement resolution, i.e., the standard deviation $\sigma_{u_{\ell}^{L}}^{e}$, is computed. $\ell=\{1,2\}$ refers to the component direction. $\sigma_{u_{\ell}^{L}}^{e}$ is defined at each 


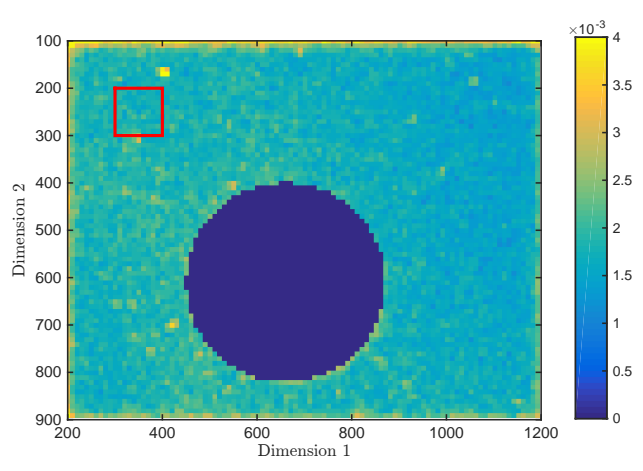

(a) Empirical vertical resolution $\sigma_{u_{2}^{\mathrm{L}}}^{e}[$ pixel]

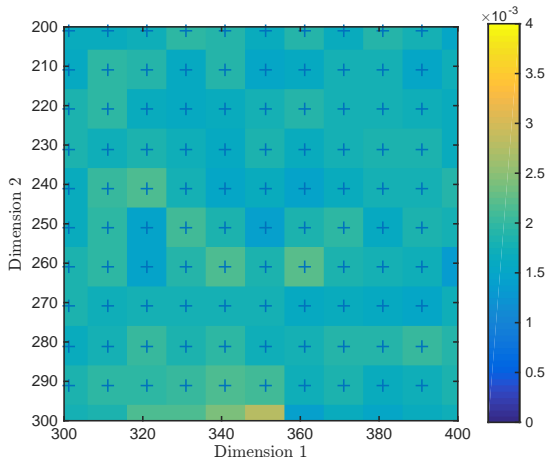

(b) Close-up (red box in (a)) [pixel]

Figure 15: (a) Observation of the vertical resolution with the L-DIC method and close-up (b). In (b), the crosses represent the center of each subset.

pixel by:

$$
\begin{aligned}
& \sigma_{u_{\ell}^{\mathrm{L}}}^{e}=\sqrt{\frac{1}{N_{\mathrm{img}}-1} \sum_{t=1}^{N_{\mathrm{img}}}\left\{\left(u_{\ell t}^{\mathrm{L}}-\left\langle u_{\ell}^{\mathrm{L}}\right\rangle\right)^{2}\right\}} \\
& \text { with }\left\langle u_{\ell}^{\mathrm{L}}\right\rangle=\frac{1}{N_{\mathrm{img}}} \sum_{t=1}^{N_{\mathrm{img}}} u_{\ell t}^{\mathrm{L}} .
\end{aligned}
$$

This vertical empirical resolution is shown in Fig.(15). Only the standard deviation $\sigma_{u_{2}^{\mathrm{L}}}^{e}$ of the

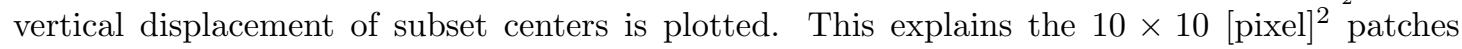
which are observable in Fig.(15b), and which correspond thus to the virtual grid composed of the subset centers.

Finally, a ratio denoted $\rho^{\mathrm{L}}$ is introduced. It is defined as the ratio between this empirical standard deviation of the vertical displacement field $\sigma_{u_{\ell}^{\mathrm{L}}}^{e}$ and the predicted one $\sigma_{u_{2}^{\mathrm{L}}}^{p}$ from Eq.(5), i.e., $\rho^{\mathrm{L}}=\frac{\sigma_{u_{2}^{\mathrm{L}}}^{e}}{\sigma_{u_{2}^{\mathrm{L}}}^{\mathrm{L}}}$. It is evaluated at each subset center.

G-DIC implementation A FE-based displacement space is also implemented using 3-node triangular elements. The mesh and a close-up are given in Fig.(16). The mesh contains 2944 elements and 1572 nodes. The displacement is thus defined through 3144 DoF. A one-step pyramidal DIC scheme is again implemented here. It uses elements which are 4 times coarser, ensuring the convergence of the G-DIC solver to the solution $\underline{u}^{\mathrm{G}}$.

Using the $N_{\text {img }}$ G-DIC realizations, an empirical displacement resolution, i.e., the standard deviation $\sigma_{u_{\ell}^{G}}^{e}$, is computed, $\ell$ referring to the component direction; i.e., $\ell=\{1,2\} . \quad \sigma_{u_{\ell}^{G}}^{e}$ is 


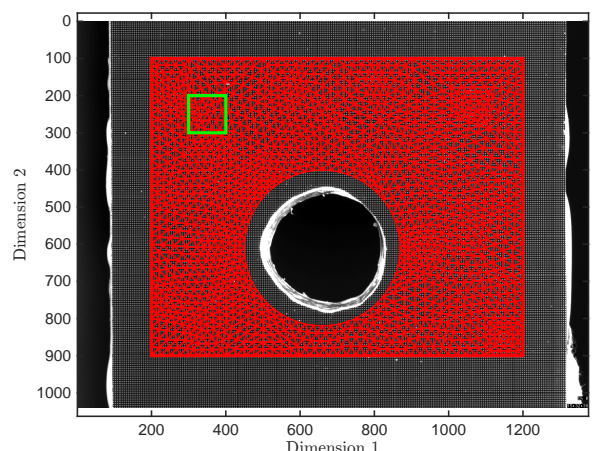

(a) RoI and mesh

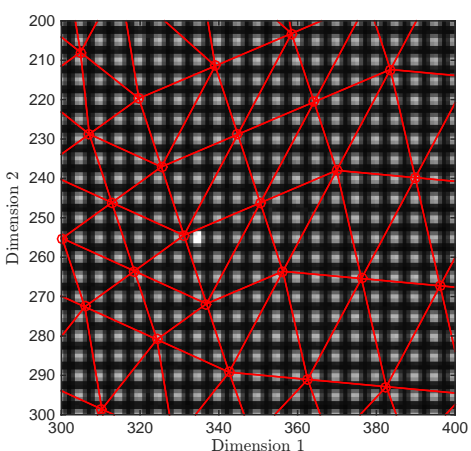

(b) Close-up (green box in (a))

Figure 16: (a) Mesh implemented for Global DIC and close-up (b).

defined at each pixel by:

$$
\begin{aligned}
& \sigma_{u_{\ell}^{\mathrm{G}}}^{e}=\sqrt{\frac{1}{N_{\mathrm{img}}-1} \sum_{t=1}^{N_{\mathrm{img}}}\left\{\left(u_{\ell t}^{\mathrm{G}}-\left\langle u_{\ell}^{\mathrm{G}}\right\rangle\right)^{2}\right\}} \\
& \text { with }\left\langle u_{\ell}^{\mathrm{G}}\right\rangle=\frac{1}{N_{\mathrm{img}}} \sum_{t=1}^{N_{\mathrm{img}}} u_{\ell t}^{\mathrm{G}} .
\end{aligned}
$$

The map of the empirical vertical displacement resolution given in Fig.(17) illustrates the spatial distribution of this ratio. The close-up in Fig.(17b) shows the variation in resolution within each element.

Finally, a ratio denoted $\rho^{\mathrm{G}}$ is introduced. It is defined as the ratio between this empirical standard deviations of the vertical displacement field $\sigma_{u_{\ell}^{\mathrm{G}}}^{e}$, and the predicted one $\sigma_{u_{2}^{\mathrm{G}}}^{p}$ given in Eq.(5); i.e., $\rho^{\mathrm{G}}=\frac{\sigma_{u_{2}^{\mathrm{G}}}^{e}}{\sigma_{u_{2}^{\mathrm{G}}}^{p}}$. It is evaluated at each nodal coordinate. To avoid any extra interpolation, nodal coordinates are also defined at pixel locations.

\subsection{Results and discussion}

Thanks to the singularity of the specimen, the spatial displacement distribution is not uniform over its surface, as illustrated in Fig.(14). Consequently, a large range of sub-pixel displacements occurs, and, according to the predictive formula Eq.(13), the resolution should be affected. Fig.(18) illustrates this phenomenon. Indeed, using the data of the GDIC case, empirical and predicted resolutions are plotted against the sub-pixel displacement. Because of the large amount of data that is available (one value per pixel), a moving average has been used instead of scattering data. Since the vertical resolution depends on both sub-pixel displacement components, the infinite norm of the sub-pixel displacement is used for Fig.(18). These first results highlight the fact that the resolution changes with the sub-pixel displacement. Nevertheless, a detailed study of this figure should go beyond simple sub-pixel dependency. Indeed, element size is non-constant over the RoI and the considered data is not reduced to only nodal data. Amongst others, these 


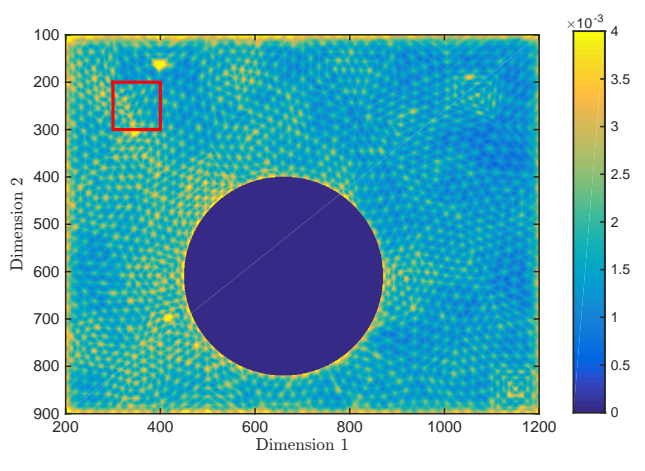

(a) Empirical vertical resolution $\sigma_{u_{\ell}^{G}}^{e}[$ pixel $]$

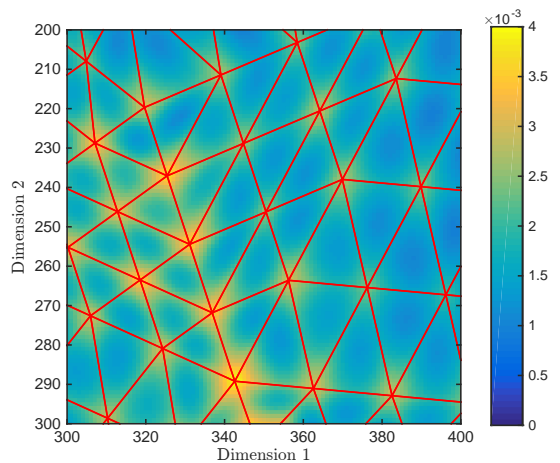

(b) Close-up (red box in (a)) [pixel]

Figure 17: (a) Observation of the vertical resolution with the FE-based G-DIC method and close-up (b).

points also affect the resolution. However, as shown in Fig.(18), the predictive formula that is proposed here correctly takes into account these effects. Both prediction and empirical resolution have here the same features.

Fig.(19) (resp. Fig.(20)) illustrates the maps and the histograms of ratio $\rho^{\mathrm{L}}$ (resp. $\rho^{\mathrm{G}}$ ). In both cases (either L-DIC or G-DIC) the ratios are equally distributed in space and their mean value is close to 1 . The spatial dispersion of the sub-pixel displacements (see Fig.(14)) is correctly taken into account in the proposed predictive formula. Moreover, both histograms also have the same general trends: their right tail is slightly more spread out. This corresponds to the pixels surrounding the RoI, which are not correctly processed. In fact, in both cases here, NRSR pre-processing is applied to the RoI, and it thus fails for border pixels because of the lack of information. Consequently, the images do not correctly remove rigid body motion. The empirical resolution is therefore higher than expected. However, it is interesting to observe that the application of the proposed formula gives the same result in both the Local and Global DIC cases. 


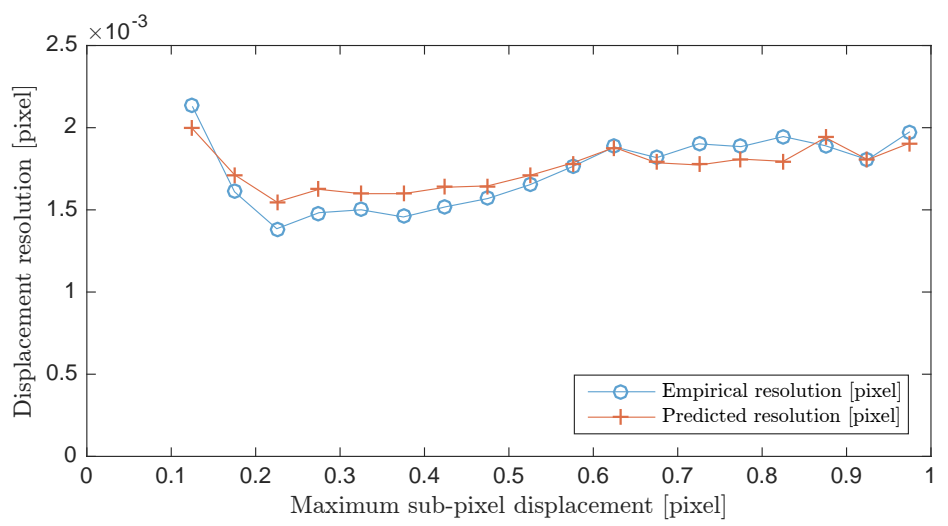

Figure 18: Observed and predicted resolution of the vertical displacement, plotted against the maximum component of the sub-pixel displacement.

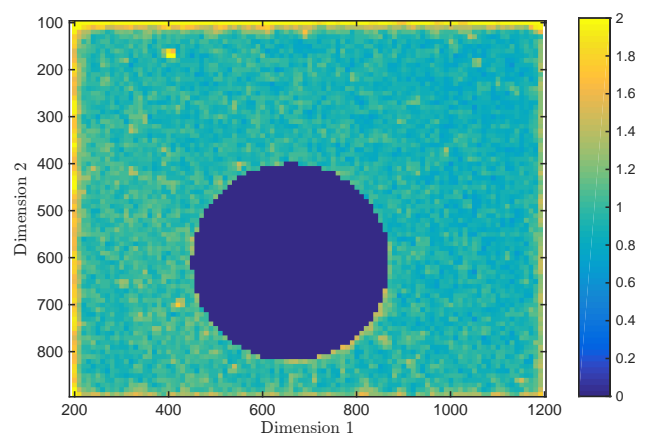

(a) Map of ratio $\rho^{\mathrm{L}}[-]$

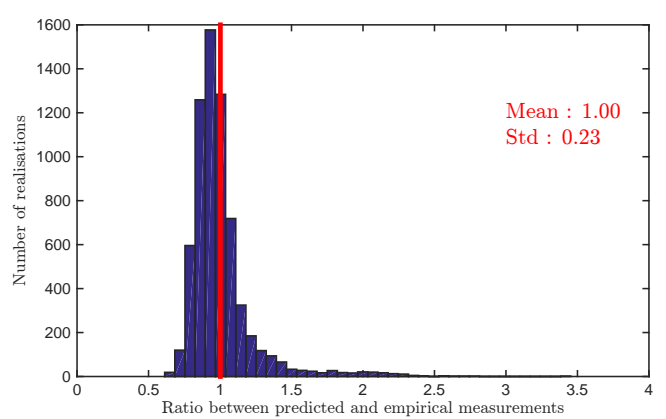

(b) Histogram of ratio $\rho^{\mathrm{L}}[-]$

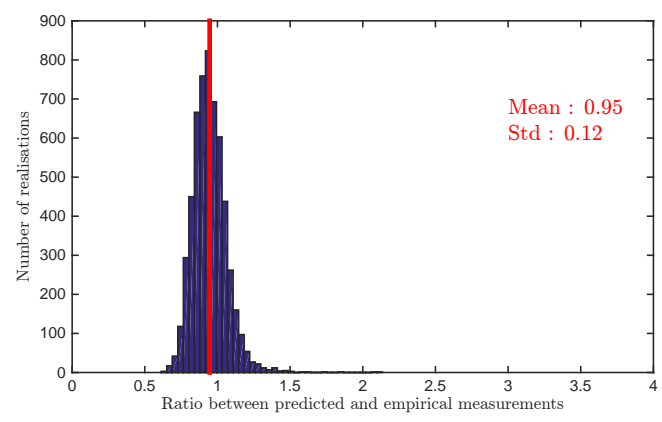

(c) Truncated histogram of ratio $\rho^{\mathrm{L}}[-]$

Figure 19: L-DIC case: Ratio between empirical and predicted vertical resolution. (a) map of the ratio; (b) histogram and (c) histogram with the border terms omitted. 


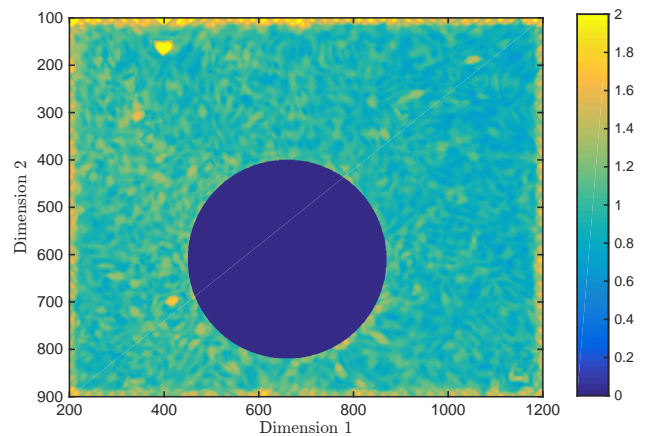

(a) Map of ratio $\rho^{\mathrm{G}}[-]$

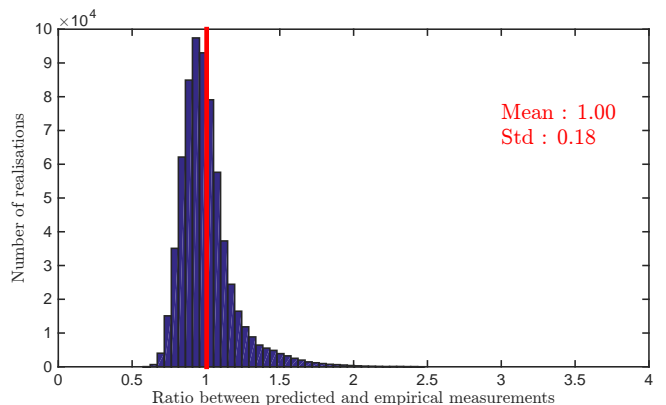

(b) Histogram of ratio $\rho^{\mathrm{G}}[-]$

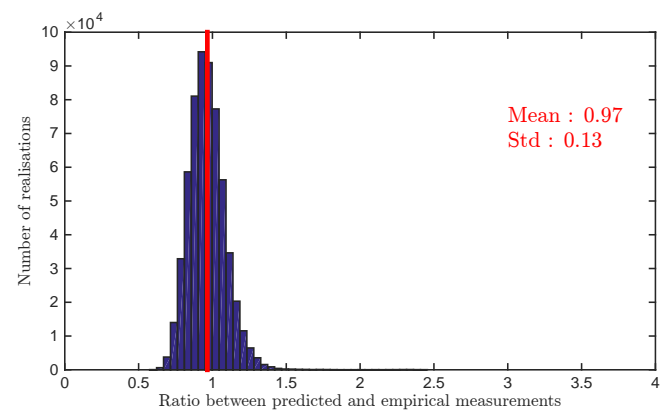

(c) Truncated histogram of ratio $\rho^{\mathrm{G}}[-]$

Figure 20: G-DIC case : Ratio between empirical and predicted vertical resolution. (a) map of the ratio; (b) histogram and (c) histogram with border terms omitted 


\section{Conclusion}

In this paper, the predictive formula for the displacement resolution obtained by DIC introduced in [4] was experimentally validated for both Global- and Local-DIC approaches. A deep study of its validation in the environment of a standard mechanical experimental laboratory was performed.

Because of this particular environment, we needed to tackle two issues: the first is the fact that camera sensor noise is not homoscedastic, as assumed so far in predictive models available in the literature, but heteroscedastic; the second is that micro-vibrations impair the characterization of noise from a stack of images.

We used specific tools to resolve these problems. We employed first the Generalized Anscombe Transform to transform heteroscedastic noise into homoscedastic noise featuring a unit standard deviation throughout the images. Another strategy was to estimate a priori pixel by pixel the distribution of the standard deviation from the images themselves, and to take this distribution into account in the prediction of the standard deviation of the noise in the displacement maps whatever their brightness, thus whatever the severity of the noise. Both led to the same results, namely a significant reduction in scatter in the pixel-by-pixel prediction of standard deviation in the displacement maps. The second problem was resolved by applying the so-called Non-Random Signal Reduction (NRSR) directly to the images to be processed. This was done before extracting the displacement fields. Since the current version of this image processing tool is available for regular markings only, we applied DIC to grid images after having preprocessed them with NRSR. Applying this second processing tool leads to the observation that experimental and predicted values for the standard deviation of the noise distribution in displacement maps are in good agreement.

An academic illustration was then discussed. It concerns a specimen subjected to a vertical translation. The obtained results emphasize the issues related to the direct application of the formula. In particular, it was necessary to account for micro-movements and noise heteroscedasticity. These data also enabled a first validation of the resolution prediction. An open-hole specimen subjected to tensile loading provided data for validation in a more realistic case, a wide range of sub-pixel displacements being covered here. Finally, it is worth noting that the generalized aspect of the formula was also demonstrated by its application to both Global- and Local-DIC techniques.

A forthcoming study will be dedicated to the introduction of a generalization of the NRSR procedure for random patterns like speckles, which is a challenging perspective.

\section{Acknowledgments}

The research group "GDR - ISIS" (CNRS) is gratefully acknowledged for its financial support (project "TIMEX").

\section{A DIC minimization scheme}

This appendix is a brief digest of the Gauss-Newton scheme in the context of DIC optimization.

\section{A.1 Notations}

We use the same notations as in Sec.(2); that is, $(f)$ and $(g)$ are the two images, $\Omega$ is the region of interest (composed of $N_{\mathrm{p}}$ pixels $\left.x_{1}, \ldots, x_{N_{\mathrm{p}}}\right), \underline{u}(\underline{x}, \boldsymbol{\lambda})$ is the displacement field at pixel $\underline{x}$, with 
the $N_{\text {DoF }} \operatorname{DoF} \boldsymbol{\lambda}=\left(\lambda_{1}, \ldots, \lambda_{N}\right)$ such that:

$$
\underline{u}(\underline{x}, \boldsymbol{\lambda})=\sum_{i=1}^{N_{\text {DoF }}} \lambda_{i} \underline{\varphi}_{i}(\underline{x})
$$

Note that $\underline{\varphi}_{i}(\underline{x})$ is a first-rank tensor, like $\underline{u}(\underline{x}, \boldsymbol{\lambda})$.

The aim of DIC-based algorithms is to find $\boldsymbol{\lambda}$ minimizing

$$
\sum_{\underline{x}_{\mathrm{p}} \in \Omega}\left(f\left(\underline{x}_{\mathrm{p}}\right)-g\left(\underline{x}_{\mathrm{p}}+\underline{u}\left(\underline{x}_{\mathrm{p}}, \boldsymbol{\lambda}\right)\right)\right)^{2}
$$

Let $r_{p}(\boldsymbol{\lambda})=f\left(\underline{x}_{\mathrm{p}}\right)-g\left(\underline{x}_{\mathrm{p}}+\underline{u}\left(\underline{x}_{\mathrm{p}}, \boldsymbol{\lambda}\right)\right)$ for any $\underline{x}_{\mathrm{p}} \in \Omega$, and $r(\boldsymbol{\lambda})=\left(r_{1}(\boldsymbol{\lambda}), \ldots, r_{N_{\mathrm{p}}}(\boldsymbol{\lambda})\right)$. Minimizing Eq.(39) is thus equivalent to minimizing the squared Euclidean norm of $\boldsymbol{r}(\boldsymbol{\lambda})$, i.e., $\|\boldsymbol{r}(\boldsymbol{\lambda})\|^{2}$.

\section{A.2 A modified Gauss-Newton method}

Let $\boldsymbol{J}_{\boldsymbol{r}}(\boldsymbol{\lambda})$ be the $N_{\mathrm{p}} \times N_{\text {DoF }}$ Jacobian matrix of $\boldsymbol{r}$ at $\lambda$. Its coefficient at row $i$ and column $j$ is:

$$
\begin{aligned}
{\left[\boldsymbol{J}_{\boldsymbol{r}}(\boldsymbol{\lambda})\right]_{i j} } & =\frac{\partial r_{i}}{\partial \lambda_{j}}(\boldsymbol{\lambda}) \\
& =\underline{\nabla} g\left(\underline{x}_{\mathrm{i}}+\underline{u}\left(\underline{x}_{\mathrm{i}}, \boldsymbol{\lambda}\right)\right) \cdot \frac{\partial \underline{u}}{\partial \lambda_{j}}\left(\underline{x}_{\mathrm{i}}, \boldsymbol{\lambda}\right) \\
& =\underline{\nabla} g^{T}\left(\underline{x}_{\mathrm{i}}+\underline{u}\left(\underline{x}_{\mathrm{i}}, \boldsymbol{\lambda}\right)\right) \cdot \underline{\varphi_{j}}\left(\underline{x}_{\mathrm{i}}\right)
\end{aligned}
$$

Eq.(42) holds from Eq.(38). Here $\underline{\nabla} g^{T}$ is the transpose of the gradient of $g$.

Assuming that $\boldsymbol{\Delta} \boldsymbol{\lambda}$ is small enough so that $\boldsymbol{r}(\boldsymbol{\lambda}+\boldsymbol{\Delta} \boldsymbol{\lambda})$ can be approximated by its first-order Taylor expansion:

$$
r(\lambda+\Delta \lambda)=r(\lambda)+J_{r}(\lambda) \Delta \lambda
$$

The Gauss-Newton algorithm consists of iterating $\boldsymbol{\lambda}^{\text {it+1 }}=\boldsymbol{\lambda}^{\text {it }}+\boldsymbol{\delta} \boldsymbol{\lambda}^{\text {it }}$ from an initial guess $\boldsymbol{\lambda}^{0}$, with $\boldsymbol{\delta} \boldsymbol{\lambda}^{\text {it }}$ minimizing $\left\|\boldsymbol{r}\left(\boldsymbol{\lambda}^{\text {it }}+\boldsymbol{\delta} \boldsymbol{\lambda}^{\text {it }}\right)\right\|^{2}$ under the approximation given by Eq.(43).

Minimizing $\left\|\boldsymbol{r}(\boldsymbol{\lambda})+\boldsymbol{J}_{\boldsymbol{r}}(\boldsymbol{\lambda}) \boldsymbol{\Delta} \boldsymbol{\lambda}\right\|^{2}$ is an ordinary least squares problem, which gives $\boldsymbol{\Delta} \boldsymbol{\lambda}$ as a solution to the normal equation:

$$
\boldsymbol{J}_{\boldsymbol{r}}(\boldsymbol{\lambda})^{T} \boldsymbol{J}_{\boldsymbol{r}}(\boldsymbol{\lambda}) \Delta \boldsymbol{\lambda}=\boldsymbol{J}_{\boldsymbol{r}}(\boldsymbol{\lambda})^{T} \boldsymbol{\lambda}
$$

This can be solved in:

$$
\boldsymbol{\Delta} \boldsymbol{\lambda}=\left(\boldsymbol{J}_{\boldsymbol{r}}(\boldsymbol{\lambda})^{T} \boldsymbol{J}_{\boldsymbol{r}}(\boldsymbol{\lambda})\right)^{-1} \boldsymbol{J}_{\boldsymbol{r}}(\boldsymbol{\lambda})^{T} \boldsymbol{r}(\boldsymbol{\lambda})
$$

Consequently,

$$
\Delta \boldsymbol{\lambda}=\widehat{\boldsymbol{M}}^{-1} \hat{\boldsymbol{b}}
$$

with

$$
\begin{aligned}
& \widehat{[\boldsymbol{M}}]_{i j}=\sum_{k}\left[\boldsymbol{J}_{\boldsymbol{r}}(\boldsymbol{\lambda})\right]_{k i}\left[\boldsymbol{J}_{\boldsymbol{r}}(\boldsymbol{\lambda})\right]_{k j}
\end{aligned}
$$

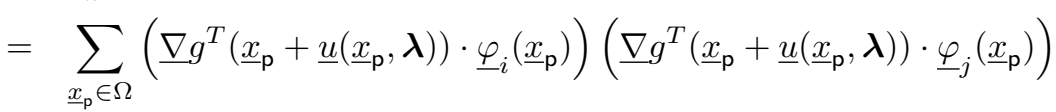


and

$$
\begin{aligned}
{[\hat{\boldsymbol{b}}]_{i} } & =\sum_{k}\left[\boldsymbol{J}_{\boldsymbol{r}}(\boldsymbol{\lambda})\right]_{k i} r_{k}(\boldsymbol{\lambda}) \\
& =\sum_{\underline{x}_{\mathrm{p}} \in \Omega}\left(\underline{\nabla} g^{T}\left(\underline{x}_{\mathrm{p}}+\underline{u}_{(}\left(\underline{x}_{\mathrm{p}}, \boldsymbol{\lambda}\right)\right) \cdot \underline{\varphi}_{i}\left(\underline{x}_{\mathrm{p}}\right)\right)\left(f\left(\underline{x}_{\mathrm{p}}\right)-g\left(\underline{x}_{\mathrm{p}}+\underline{u}_{\left.\left.\left(\underline{x}_{\mathrm{p}}, \underline{\lambda}\right)\right)\right)}\right.\right.
\end{aligned}
$$

However, the term $\underline{\nabla} g\left(\underline{x}_{\mathrm{p}}+\underline{u}\left(\underline{x}_{\mathrm{p}}, \underline{\lambda}\right)\right)$ necessitates a numerical interpolation scheme, since $g$ is known only on the pixel grid. Instead of repeating this procedure for each iteration, it is more convenient to identify $\underline{\nabla} g\left(\underline{x}_{\mathrm{p}}+\underline{u}\left(\underline{x}_{\mathrm{p}}, \underline{\lambda}\right)\right)$ with $\underline{\nabla} f\left(\underline{x}_{\mathrm{p}}\right)$, which is a licit approximation when $\boldsymbol{\lambda}$ is close to minimizing Eq.(39) if a small displacement hypothesis is assumed (cf [25]).

With this modified Gauss-Newton scheme, the following formula holds:

$$
\Delta \lambda=M^{-1} b
$$

with

$$
[\boldsymbol{M}]_{i j}=\sum_{\underline{x}_{\mathrm{p}} \in \Omega}\left(\underline{\nabla} f^{T}\left(\underline{x}_{\mathrm{p}}\right) \cdot \underline{\varphi}_{i}\left(\underline{x}_{\mathrm{p}}\right)\right)\left(\underline{\nabla} f^{T}\left(\underline{x}_{\mathrm{p}}\right) \cdot \varphi_{j}\left(\underline{x}_{\mathrm{p}}\right)\right)
$$

and

$$
[\boldsymbol{b}]_{i}=\sum_{\underline{x}_{\mathrm{p}} \in \Omega}\left(\underline{\nabla} f^{T}\left(\underline{x}_{\mathrm{p}}\right) \cdot \underline{\varphi}_{i}\left(\underline{x}_{\mathrm{p}}\right)\right)\left(f\left(\underline{x}_{\mathrm{p}}\right)-g\left(\underline{x}_{\mathrm{p}}+\underline{u}\left(\underline{x}_{\mathrm{p}}, \boldsymbol{\lambda}\right)\right)\right)
$$

Note that $\boldsymbol{M}$ is a symmetrical matrix.

\section{B DIC on a grid}

A grid was deposited using the procedure described in [33] on a similar specimen as the one in Section 3, as illustrated in Fig.(21a). The same experimental set-up was used and the same experimental methodology was applied. In order to allow statistical analysis, $N_{\mathrm{img}}=100$ pictures were also acquired at each step of the tests, i.e., $\left(\left(\mathrm{f}_{t}\right)_{1 \leq t \leq N_{\mathrm{img}}}\right)$ describing the reference state and $\left(\left(\mathrm{g}_{t}\right)_{1 \leq t \leq N_{\text {img }}}\right)$ the current one. Fig.(21a) illustrates the grid (f), which was used throughout this paper. Fig.(21b) presents a close-up of this grid; the lines are clearly visible, and the pitch of 5 pixels can be distinguished. Because the grid used in this study is composed of black lines over a white background, it presents darker intensity levels than for a usual speckle, as used in the study above. It is clearly visible in Fig.(21a), which can be compared with the speckle, $c f$ Fig.(1b).

The main difficulty in applying DIC to a grid is the initial guess that should be made to initiate the method. This difficulty can easily be overcome using a pyramidal DIC approach, as introduced in [10]. Here, the few defects impairing the grid drive the DIC solution at the coarsest scales towards the right solution and thus feed the final fine DIC solving correctly. This initialization functions work correctly in both examples illustrating this article, i.e., the vertical translation and the open-hole problem. The finest DIC mesh is plotted over the grid in Fig.(21b), highlighting the arbitrary choice for the element size of $13 \times 13$ pixels. Such a choice thus ensures a mismatch between the support of each nodal function, and by consequence between each local residual that the DIC method minimizes $(c f \mathrm{~A})$.

Fig.(22) summarizes the main statistical characteristics associated with the grid images that were acquired. The maps of the average pixel value $\langle f\rangle$ ( $c f$ Fig.(22a), close-up Fig.(22c)) and of the standard deviation $\sigma_{f}$ (cf Fig.(22b), close-up Fig.(22d)) are shown. The heteroscedatic nature of this noise is clearly visible in Fig.(22b \& d), in which the blue lines (from Fig.(22d), 


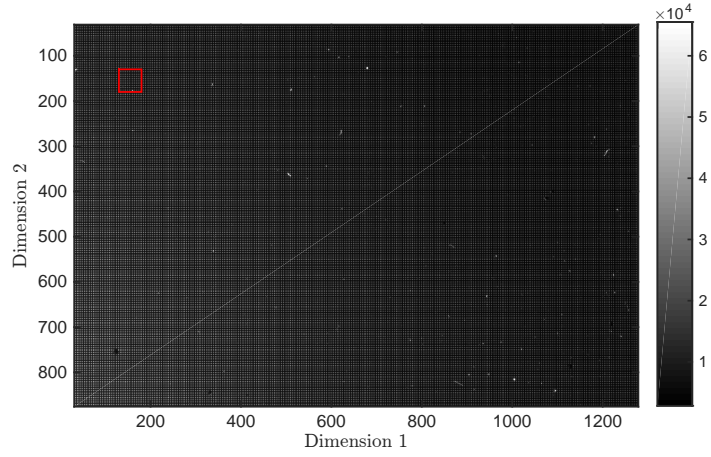

(a) Region of Interest [gray level]

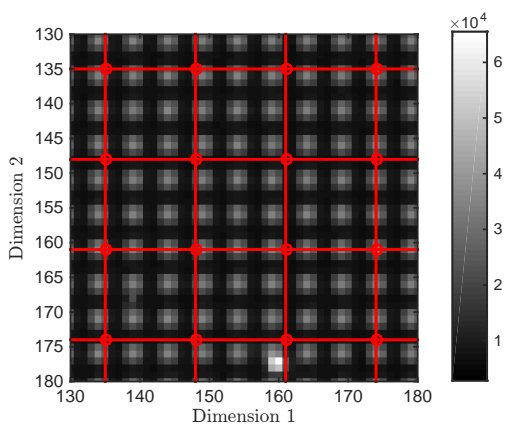

(b) Close-up of the RoI (red box in (b)) [gray level]

Figure 21: Illustration of the imaged grid. (a) Image of the grid; the red box specifies the location of the close-up. (b) Close-up of one spot of the RoI and the mesh plotted on top of it, illustrating the regular definition of the grid pattern.

which correspond to low standard deviation) correspond to the black lines of the grid (which are of low light intensity, $c f$ Fig.(22b)).

A DIC calculation is carried out using the same displacement description as in Section 3.1. On the one hand, the formula in Eq.(13) can of course be applied here, and we obtain the predicted standard deviation at each node. This is depicted in Fig.(23a). The ratio between predicted measurements of the displacement standard deviation when DIC is applied to a grid or a random speckle is also presented in Fig.(23b). This ratio lying between 0 and 1, it a priori highlights interesting properties for applying DIC to grids. This is due to the higher values of the image gradient of a grid than of usual speckles like that used in this study. Since the square of the image gradient is involved in the correlation matrix $\boldsymbol{M}$, and since predicted formulas are based on $\boldsymbol{M}^{-1}$, higher gradients lead to a lower displacement resolution standard deviation.

On the other hand, Fig.(24) illustrates the empirical displacement standard deviation. Indeed, since $N_{\text {img }}$ images were acquired at both time steps, the reference image and the current one, $N_{\text {img }}$ DIC calculations can be performed, and, as in Section 3, a statistical analysis can also be performed on the DIC outputs. Fig.(24a) thus maps the observed displacement standard deviation $\sigma_{\mathrm{u}_{2}}^{p}$ (only the vertical component is mapped) whereas Fig.(24b) illustrates the ratio between observed resolutions when DIC is applied to a grid or to a speckle. The ratio here is higher than with the predictive formulas, but is still slightly smaller than 1.

As a conclusion to this pre-study, we can say that applying DIC to a grid image does not preset any drawbacks, provided that the DIC algorithm has been adequately initialized. Moreover, the particularly high gradients that are present in the grid images can offer interesting properties in terms of mastering displacement resolution.

Finally, Fig. (25) presents the ratio between the empirical and predicted displacement resolution. As with the speckle-based pattern, a heterogeneous spatial distribution of the ratio is visible in Fig.(25a), and its mean value is greater than 1. There is an underestimation of the actual measurement resolution, which is due to both the RBM that occurs between the camera and the specimen, and to the assumed homoscedasticity hypothesis for the image noise. 


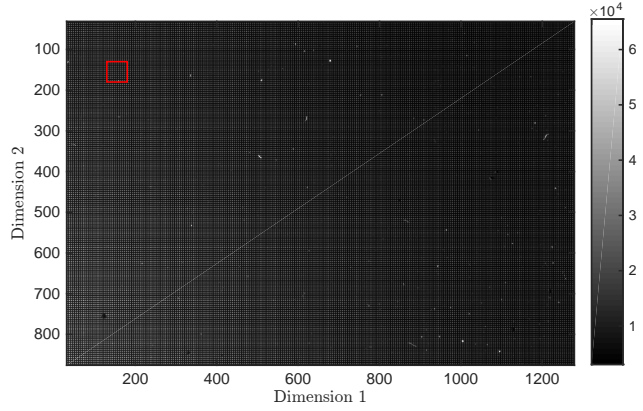

(a) Pixel mean value $\langle\mathrm{f}\rangle$ [gray level]

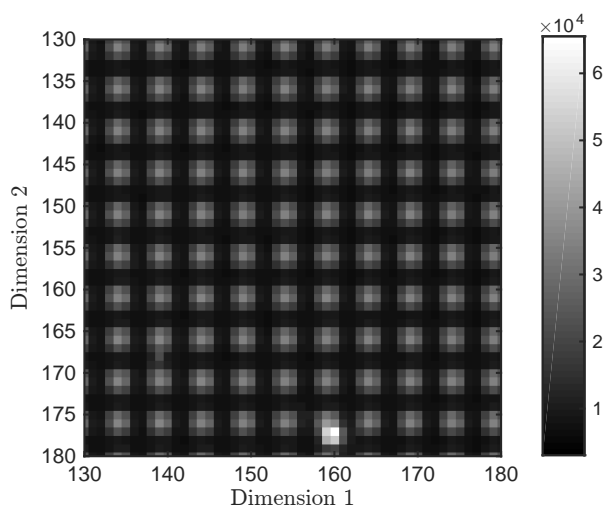

(c) Close-up - pixel mean value $\langle\mathrm{f}\rangle$ [gray level]

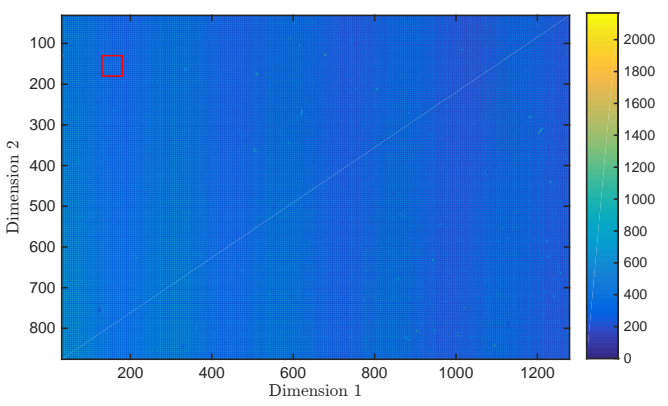

(b) Pixel standard deviation $\sigma_{\mathrm{f}, \mathrm{p}}$ [gray level]

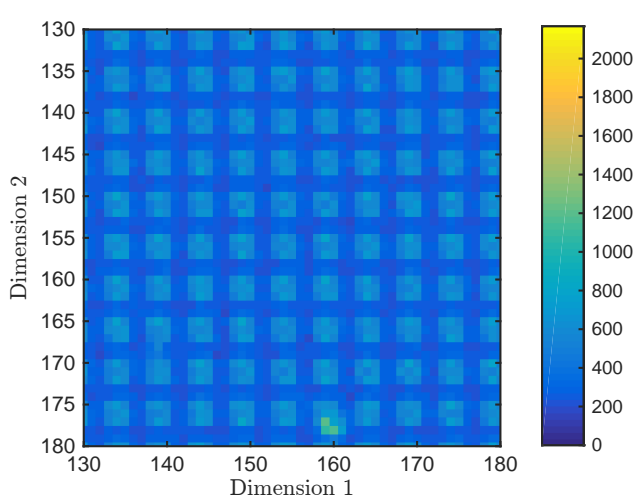

(d) Close-up - pixel standard deviation $\sigma_{\mathrm{f}, \mathrm{p}}$ [gray level]

Figure 22: Main characteristics of the statistical analysis applied to grid images, namely the pixel mean value $\langle\mathrm{f}\rangle$ (a), the pixel standard deviation $\sigma_{\mathrm{f}, \mathrm{p}}(\mathrm{b})$, and two close-ups of $\langle\mathrm{f}\rangle$ (c) and $\sigma_{\mathrm{f}, \mathrm{p}}(\mathrm{d})$. 


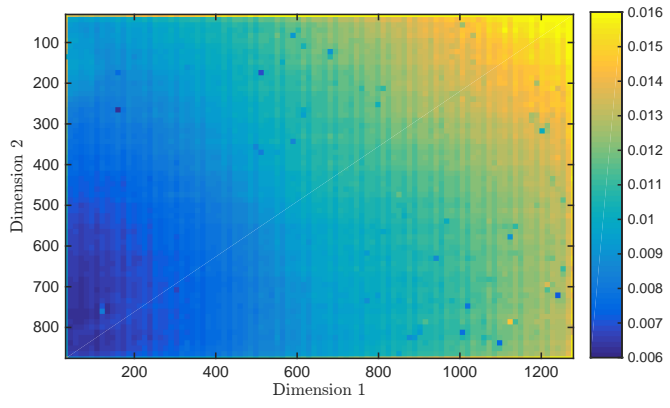

(a) Standard deviation $\left(\sigma_{\mathrm{u}_{2}}^{p}\right)$ [pixel]

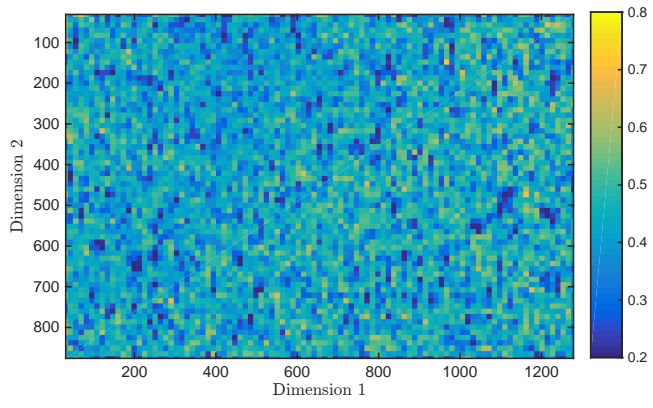

(b) Grid/Speckle ratio $\left(\sigma_{\mathrm{u}_{2}}^{p} / \sigma_{u_{2}}^{p}\right)[-]$

Figure 23: Predicted standard deviation of the vertical displacement $\left(\sigma_{\mathrm{u}_{2}}^{p}\right)$ and its ratio to that obtained when DIC is applied to the speckle.

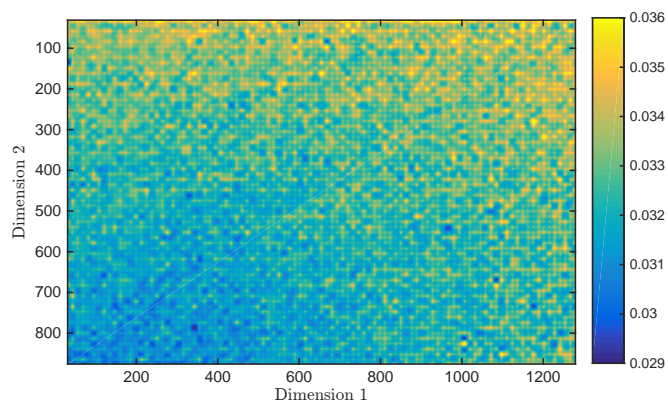

(a) Standard deviation $\left(\sigma_{\mathrm{u}_{2}}^{e}\right)$ [pixel]

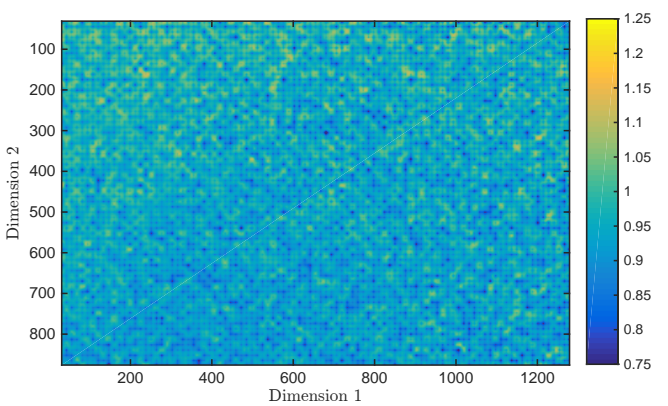

(b) Grid/Speckle ratio $\left(\sigma_{\mathrm{u}_{2}}^{e} / \sigma_{u_{2}}^{e}\right)[-]$

Figure 24: Empirical standard deviation of the vertical displacement $\left(\sigma_{\mathrm{u}_{2}}^{e}\right)$ and its ratio to that obtained when DIC is applied to the speckle 


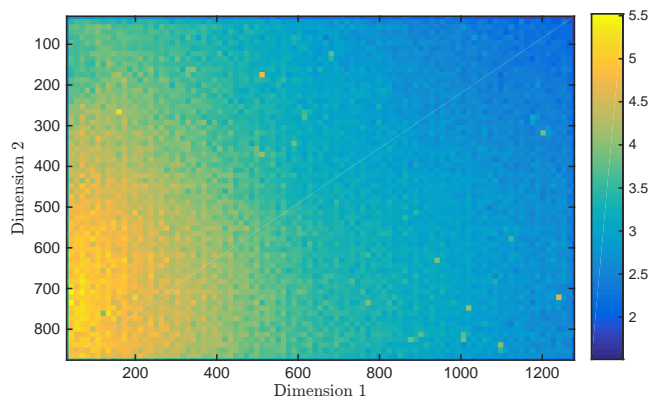

(a) Map of ratio $\rho_{\mathrm{u}}[-]$

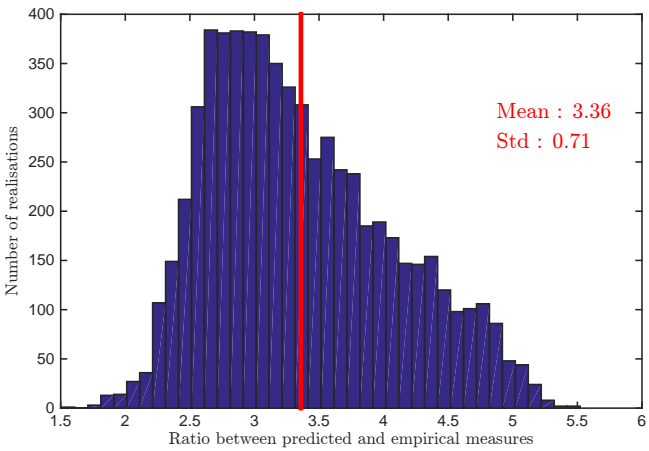

(b) Histogram of ratio $\rho_{\mathrm{u}}[-]$

Figure 25: Ratio between observed and predicted standard deviation of the vertical displacement, $\rho_{\mathrm{u}}=\sigma_{\mathrm{u}_{2}}^{e} / \sigma_{\mathrm{u}_{2}}^{p}$.

\section{References}

[1] F. Anscombe. The transformation of Poisson binomial and negative-binomial data. Biometrika, 35(3-4):246-254, 1948.

[2] G. Besnard, F. Hild, and S. Roux. Finite-element displacement fields analysis from digital images: application to Portevin-Le Châtelier bands. Experimental Mechanics, 46(6):789803, 2006.

[3] B. Blaysat, E. Florentin, G. Lubineau, and A. Moussawi. A dissipation gap method for fullfield measurement-based identification of elasto-plastic material parameters. International Journal for Numerical Methods in Engineering, 91(7):685-704, 2012.

[4] B. Blaysat, M. Grédiac, and F. Sur. Effect of interpolation on noise propagation from images to DIC displacement maps. International Journal for Numerical Methods in Engineering, Avalaible online (doi:v10.1002/nme.5212), 2016.

[5] B. Blaysat, J. P. M. Hoefnagels, G. Lubineau, M. Alfano, and M. G. D. Geers. Interface debonding characterization by image correlation integrated with double cantilever beam kinematics. International Journal of Solids and Structures, 55:79-91, 2015.

[6] S. Bossuyt. Optimized patterns for digital image correlation. In Proceedings of the 2012 Annual Conference on Experimental and Applied Mechanics, volume 3: Imaging Methods for Novel Materials and Challenging Applications, pages 239-248. Springer, 2013.

[7] J. Boulanger, C. Kervrann, P. Bouthemy, P. Elbau, J.-B. Sibarita, and J. Salamero. Patchbased nonlocal functional for denoising fluorescence microscopy image sequences. IEEE Transactions on Medical Imaging, 29(2):442-454, 2010.

[8] T. C. Chu, W. F. Ranson, and M. A. Sutton. Applications of Digital-Image-Correlation techniques to experimental mechanics. Experimental Mechanics, 25(3):232-244, 1985.

[9] X. Fayolle and F. Hild. Controlling stress intensity factor histories with digital images. Experimental Mechanics, 54(2):305-314, 2014. 
[10] R. Fedele, L. Galantucci, and A. Ciani. Global 2D digital image correlation for motion estimation in a finite element framework: a variational formulation and a regularized, pyramidal, multi-grid implementation. International Journal for Numerical Methods in Engineering, 96(12):739-762, 2013.

[11] A. Foi, M. Trimeche, V. Katkovnik, and K. Egiazarian. Practical Poissonian-Gaussian noise modeling and fitting for single-image raw-data. IEEE Transactions on Image Processing, 17(10):1737-1754, 2008.

[12] R. Gras, H. Leclerc, F. Hild, S. Roux, and J. Schneider. Identification of a set of macroscopic elastic parameters in a 3D woven composite: Uncertainty analysis and regularization. International Journal of Solids and Structures, 55:2-16, 2015.

[13] M. Grédiac and F. Hild. Full-field measurements and identification in solid mechanics. Wiley, 2013.

[14] M. Grédiac and F. Sur. Effect of sensor noise on the resolution and spatial resolution of displacement and strain maps estimated with the grid method. Strain, 50(1):1-27, 2014.

[15] A. Gut. An Intermediate Course in Probability. Springer, 2009.

[16] G. Healey and R. Kondepudy. Radiometric CCD camera calibration and noise estimation. IEEE Transactions on Pattern Analysis and Machine Intelligence, 16(3):267-276, 1994.

[17] F. Hild and S. Roux. Digital Image Correlation: An experimental mechanics tool. LMTCachan. Internal report.

[18] F. Hild and S. Roux. Comparison of local and global approaches to digital image correlation. Experimental Mechanics, 52:1503-1519, 2012.

[19] G. C. Holst. CCD Arrays Cameras and Displays. JCD Publishing, 1998.

[20] JCGM Member Organizations. International vocabulary of metrology - Basic and general concepts and associated terms (VIM), volume 200. BIPM, 2012.

[21] X. D. Ke, H. W. Schreier, M. A. Sutton, and Y. Q. Wang. On error assessment in stereobased deformation measurements. Experimental Mechanics, 51(4):423-441, 2011.

[22] D. Lecompte, A. Smits, S. Bossuyt, H. Sol, J. Vantomme, D. V. Hemelrijck, and A. Habraken. Quality assessment of speckle patterns for digital image correlation. Optics and Lasers in Engineering, 44(11):1132 - 1145, 2006.

[23] F. Mathieu, H. Leclerc, F. Hild, and S. Roux. Estimation of elastoplastic parameters via weighted FEMU and Integrated-DIC. Experimental Mechanics, 55(1):105-119, 2015.

[24] F. Murtagh, J.-L. Starck, and A. Bijaoui. Image restoration with noise suppression using a multiresolution support. Astronomy and Astrophysics, Suppl. Ser, 112:179-189, 1995.

[25] J. Neggers, B. Blaysat, J. P. M. Hoefnagels, and M. G. D. Geers. On image gradients in digital image correlation. International Journal for Numerical Methods in Engineering, 105(4):243 - 260, 2016.

[26] B. Pan, B. Wang, G. Lubineau, and A. Moussawi. Comparison of subset-based local and finite element-based global digital image correlation. Experimental Mechanics, pages 1-15, 2015 . 
[27] B. Pan, H. Xie, and Z. Wang. Equivalence of digital image correlation criteria for pattern matching. Applied Optics, 49(28):5501-5509, 2010.

[28] B. Pan, H. Xie, Z. Wang, K. Qian, and Z. Wang. Study on subset size selection in digital image correlation for speckle patterns. Optics Express, 16(10):7037-7048, 2008.

[29] B. Pan, L. Yu, and D. Wu. High-accuracy 2d digital image correlation measurements with bilateral telecentric lenses: Error analysis and experimental verification. Experimental Mechanics, 53(9):1719-1733, 2013.

[30] B. Pan, L. Yu, D. Wu, and L. Tang. Systematic errors in two-dimensional digital image correlation due to lens distortion. Optics and Lasers in Engineering, 51(2):140-147, 22013.

[31] J.-C. Passieux, F. Bugarin, C. David, J.-N. Périé, and L. Robert. Multiscale displacement field measurement using digital image correlation: Application to the identification of elastic properties. Experimental Mechanics, 55(1):121-137, 2015.

[32] J.-C. Passieux, J. N. Périé, and M. Salaün. A dual domain decomposition method for finite element digital image correlation. International Journal for Numerical Methods in Engineering, 102(10):1670-1682, 2015.

[33] J. L. Piro and M. Grédiac. Producing and transferring low-spatial-frequency grids for measuring displacement fields with moiré and grid methods. Experimental Techniques, 28(4):23$26,2004$.

[34] J. Réthoré. A fully integrated noise robust strategy for the identification of constitutive laws from digital images. International Journal for Numerical Methods in Engineering, 84(6):631-660, 2010.

[35] J. Réthoré, G. Besnard, G. Vivier, F. Hild, and S. Roux. Experimental investigation of localized phenomena using digital image correlation. Philosophical Magazine, 88(28-29):3339$3355,2008$.

[36] J. Réthoré, F. Hild, and S. Roux. Extended digital image correlation with crack shape optimization. International Journal for Numerical Methods in Engineering, 73(2):248-272, 2008.

[37] J. Réthoré, S. Roux, and F. Hild. An extended and integrated digital image correlation technique applied to the analysis of fractured samples. European Journal of Computational Mechanics, 18(3-4):285-306, 2009.

[38] J. Réthoré, S. Roux, and F. Hild. Optimal and noise-robust extraction of fracture mechanics parameters from kinematic measurements. Engineering Fracture Mechanics, 78(9):1827 $1845,2011$.

[39] S. Roux and F. Hild. Stress intensity factor measurements from digital image correlation: post-processing and integrated approaches. International Journal of Fracture, 140:141-157, 2006.

[40] H. Schreier, J.-J. Orteu, and M. A. Sutton. Image correlation for shape, motion and deformation measurements: Basic Concepts, Theory and Applications. Springer, 2009.

[41] F. Sur and M. Grédiac. Sensor noise modeling by stacking pseudo-periodic grid images affected by vibrations. IEEE Signal Processing Letters, 21(4):432-436, 2014. 
[42] F. Sur and M. Grédiac. Measuring the noise of digital imaging sensors by stacking raw images affected by vibrations and illumination flickering. SIAM Journal on Imaging Sciences, 8(1):611-643, 2015.

[43] F. Sur and M. Grédiac. On noise reduction in strain maps obtained with the grid method by averaging images affected by vibrations. Optics and Lasers in Engineering, 66:210-222, 2015.

[44] F. Sur, N. Noury, and M.-O. Berger. Computing the uncertainty of the 8 point algorithm for fundamental matrix estimation. In Proceedings of the British Machine Vision Conference (BMVC), volume 2, pages 965-974, Leeds (UK), September 2008.

[45] M. Sutton, J. Yan, V. Tiwari, H. Schreier, and J. Orteu. The effect of out-of-plane motion on $2 \mathrm{~d}$ and $3 \mathrm{~d}$ digital image correlation measurements. Optics and Lasers in Engineering, 46(10):746 - 757, 2008.

[46] M. A. Sutton, C. Mingqi, W. H. Peters, Y. J. Chao, and S. R. McNeill. Application of an optimized digital correlation method to planar deformation analysis. Image and Vision Computing, 4(3):143 - 150, 1986.

[47] W. Tong. Reduction of noise-induced bias in displacement estimation by linear off-pixel digital image correlation. Strain, 49(2):158-166, 2013.

[48] Y. Q. Wang, M. A. Sutton, H. A. Bruck, and H. W. Schreier. Quantitative error assessment in pattern matching: Effects of intensity pattern noise, interpolation, strain and image contrast on motion measurements. Strain, 45(2):160-178, 2009.

[49] Y. Q. Wang, M. A. Sutton, X. D. Ke, H. W. Schreier, P. L. Reu, and T. J. Miller. On error assessment in stereo-based deformation measurements. Experimental Mechanics, 51(4):405$422,2011$.

[50] Z. Y. Wang, H. Q. Li, J. W. Tong, and J. T. Ruan. Statistical analysis of the effect of intensity pattern noise on the displacement measurement precision of digital image correlation using self-correlated images. Experimental Mechanics, 47(5):701-707, 2007.

[51] L. Wittevrongel, P. Lava, S. V. Lomov, and D. Debruyne. A self adaptive global digital image correlation algorithm. Experimental Mechanics, 55(2):361-378, 2015.

[52] S. Yoneyama, H. Kikuta, A. Kitagawa, and K. Kitamura. Lens distortion correction for digital image correlation by measuring rigid body displacement. Optical Engineering, 45(2):023602, 2006. 